\title{
Gestión de recursos humanos en una institución pública. Un modelo conjunto de los sistemas de clasificación profesional, carrera profesional y de retribuciones
}

\author{
Ana Ma Vázquez Losada \\ Consejo Económico y Social \\ ana.vazquez@ces.es
}

\begin{abstract}
Resumen
El estudio realizado tiene por objeto presentar los sistemas de clasificación profesional, carrera profesional y de retribuciones para el personal laboral de un organismo público, cuya ley de creación lo configura como un Ente de Derecho Público con plena autonomía orgánica y funcional para el cumplimiento de sus fines. A partir de la información contenida en el organigrama de la institución y en un informe elaborado para ésta por la Consultoría Operativa de Servicios del Ministerio de Administraciones Públicas, se realiza un análisis de funciones que da lugar a quince puestos tipo que se ordenan en grupos, niveles y categorías profesionales, constituyendo, así, el cuadro de clasificación profesional. Aplicando una metodología de Análisis y Valoración de Puestos, se determinan los coeficientes de clasificación de nivel $(\alpha)$, categoría $(\beta)$, puesto tipo $(\gamma)$ y de carrera horizontal $(\delta)$ que son la base sobre la que se sustentan los sistemas de promoción horizontal y vertical y el retributivo que se proponen. La principal ventaja de este método es que los coeficientes tienen carácter atemporal, lo que significa que una vez determinado el valor de cada uno de ellos, no tendrían que modificarse.
\end{abstract}

Palabras clave Carrera administrativa, clasificación profesional, empleado público, sistema retributivo.

\section{Human resources management in a public institution. An integrated model of professional classification, career and remuneration systems}

\begin{abstract}
This article aims to present the systems of professional classification, professional career and remuneration for the personnel of a public organism whose law of creation forms it as an Entity of Public law with organic and functional autonomy. From the information contained in the flowchart of the institution and in a report elaborated for this one by the Operative Consultancy of Services of the Department of Public Administrations there is realized an analysis of functions that gives place to fifteen positions type which are ordained in groups, levels and professional categories, constituting this way the picture of professional classification. Applying a methodology of Analysis and valuation of Positions, the coefficients of level classification ( $\alpha$ ), category ( $(B)$, position type $(\gamma)$ and of horizontal career $(\delta)$, that are the base on which there are sustained the systems of horizontal and vertical promotion and the remunerative one that they propose. The principal advantage of this method is that the values of the coefficients have character atemporal what means that once determined the value of each one of them, would not have to be modified.
\end{abstract}

Keywords

Administrative career, professional classification, civil servant, remuneration system. 


\section{OBJETO Y JUSTIFICACIÓN}

Cualquier organización que quiera implantar un modelo de gestión de recursos humanos tiene que diseñar o rediseñar los procesos y herramientas referidas a organización, valoración, análisis de puestos, selección, formación, carrera y evaluación de desempeño.

Si se trata de una institución pública, hay que acomodar, además, el uso de esos procesos y herramientas a las especificaciones legales que regulen los diferentes ámbitos materiales de actuación.

En este aspecto destaca, por su centralidad, la Ley $7 / 2007$, de 7 de abril, del Estatuto Básico del Empleado Público (EBEP) que proporciona el marco general para la gestión de recursos humanos en las administraciones públicas.

Este es el punto de partida de este estudio cuyo objeto es presentar los sistemas de clasificación profesional, carrera profesional y retribuciones para el personal de un organismo público de carácter consultivo, cuya ley de creación lo configura como un Ente de Derecho Público con plena autonomía orgánica y funcional para el cumplimiento de sus fines y con una plantilla de cien empleados, contratados laborales.

Se aborda la cuestión de una manera integral de forma que se puedan establecer y regular las interconexiones existentes entre los tres sistemas mencionados.

A la hora de abordar esta propuesta se ha tenido en cuenta, desde el punto de vista metodológico: 1) la identificación y clasificación de las actividades de la organización, 2) la agrupación de las actividades necesarias para el cumplimiento de los objetivos, 3) la asignación de cada grupo de actividades a un profesional dotado de la autoridad necesaria para supervisarlo y 4) la estipulación de coordinación horizontal y vertical en la estructura organizacional (Chiavenato, 1999; Koontz y Weihrich, 1990; Stoner et al., 1996).

El trabajo consta de cinco apartados. En el primero se hace una exposición general del marco teórico, el segundo se dedica al sistema de clasificación profesional que va a permitir comprender la formulación de los que se presentan después para la carrera administrativa y el sistema retributivo que se abordan en el tercero y cuarto. En el quinto se exponen las conclusiones.

\section{MARCO TEÓRICO Y LEGAL}

El EBEP constituye una norma de mínimos que intenta integrar en un único texto legal, de alcance general, el régimen aplicable a todo el personal al servicio de las administraciones públicas. Pero esa norma no ha unificado de forma absoluta los dos

12 regímenes jurídicos, funcionarial y laboral, sino que ha generado un "núcleo regula- 
dor común" manteniendo las especialidades y, por tanto, la dualidad reguladora, en lo que no es susceptible de integración (Molina Navarrete, 2007: 127).

Para el personal laboral no es un estatuto propiamente dicho, ni siquiera una norma de mínimos ya que en la conformación del modelo se hace concurrente y primaria tanto la aplicación de la legislación laboral, y las demás normas convencionalmente aplicables, como los preceptos que así lo dispongan (art. 7 EBEP).

Desde el punto de vista de su alcance subjetivo, una innovación destacable del Estatuto es su extensión a las "entidades de derecho público con personalidad jurídica propia, vinculadas o dependientes de cualquiera de las Administraciones Públicas". Se produce así una ampliación considerable respecto a la situación precedente por cuanto el régimen jurídico del empleo público determinado por Ley 30/1984, de 2 de agosto, de Medidas para la Reforma de la función Pública y por el art. 45 de la Ley 6/1997, de 14 de abril, de Organización y Funcionamiento de la Administración General del Estado, quedaba circunscrito básicamente al personal de los organismos públicos que se rigen por el Derecho administrativo (Cantero Martínez, 2007: 59).

Sobre el carácter de la relación laboral, aparece un problema ya surgido en el pasado (Godino Reyes, 1996: 279 y ss.) que posee una relevancia casi exclusivamente formal. Es el relativo a si la relación de los empleados públicos laborales debe configurarse o no como una relación laboral especial. Un sector de la doctrina científica se muestra a favor de considerarlo como una relación laboral especial en virtud de las múltiples peculiaridades que se regulan en el EBEP (Sala Franco, 2007: 117; Cantero Martínez, 2007: 97); otro, sin embargo, apelando a la dicción del art. 2, 1.i) del Estatuto de los Trabajadores, se inclina por conceptuarlo como un vínculo común con peculiaridades (Landa Zapirain, 2008: 2).

La clasificación profesional distribuye a los trabajadores en función de diversos niveles profesionales y el criterio de jerarquización gira en torno a la profesionalización (Edwards, 1990; Castillo Mendoza, 1990: 117-140).

En esta materia, el art. 77 del EBEP establece que "el personal laboral se clasificará de conformidad con la legislación laboral”. En tal sentido, el art. 22.1 del Estatuto de los Trabajadores (ETT) obliga a disponer un sistema de clasificación profesional que se establecerá “mediante la negociación colectiva o, en su defecto, por acuerdo entre la empresa y los representantes de los trabajadores, por medio de categorías o grupos profesionales".

En lo que respecta a la promoción y carrera administrativa, la doctrina, en su mayoría, viene aceptando el concepto de movilidad funcional como el cambio de las funciones habitualmente prestadas por el trabajador, con o sin cambio del puesto de trabajo (Martínez Abascal, 1994: 762; Sala Franco, 1998: 5-7). Cuando esta movilidad se realiza en el sentido ascendente, se habla de promoción profesional y 
ha de realizarse cumpliendo las normas convencionales que regulan los ascensos (Alia Ramos y Montane Marinero: 1995: 59; Sala Franco y López Tarruella Martínez, 1991: 40).

Ello significa que la promoción ha de ajustarse a una serie de principios y limitaciones $^{1}$ y a las reglas jurídicas que, en protección o beneficio de los trabajadores, no pueden ser transgredidas por ningún cambio funcional (Alia Ramos y Montane Merinero, 1995: 149; Cruz Villalón, 1983: 377).

El EBEP ha incorporado una regulación innovadora y novedosa al permitir que se articulen modelos de carrera horizontal desvinculada de los cambios de puesto de trabajo, basada en el desarrollo de las competencias y en el rendimiento. Establece varias modalidades de promoción aplicables, en principio, para el personal funcionario, pero nada impide su aplicación también al personal laboral para el cual el propio texto estatutario regula, en su artículo 19, la promoción profesional como un derecho de los empleados públicos. Si bien hay que señalar que el régimen de carrera para personal laboral y funcionarios exige no solo procedimientos distintos, sino también cauces e instrumentos jurídicos diferentes.

En el EBEP, el derecho a la carrera va a ser un auténtico derecho subjetivo funcional que se inicia con la toma de posesión del primer destino o puesto de trabajo, y que se plasma en la posibilidad de progresar de forma ascendente e ininterrumpida a lo largo de su vida profesional. Dos son los principios inspiradores de la misma: el de gradualidad o progresión y el de consolidación o irreversibilidad (Manzana Laguarda, 2006: 233-234).

La carrera, pues, tiene su proyección sobre dos aspectos: una mejor retribución y una mayor cualificación. Su fundamento entronca directamente con los principios de eficacia, jerarquía y promoción a través del trabajo².

Pero junto a esta dimensión de la carrera como derecho individual, se presenta una dimensión institucional que, como indica Férez Fernández, es "la otra cara de la moneda" y como señala Palomar Olmeda, "el derecho de opción del modelo se encuentra limitado en las modalidades de carrera pero no en la intensidad de las mismas

1 La exigencia de la titulación o de aptitud necesaria para el desarrollo de la función asignada, la dignidad del trabajador, la buena fe, la prohibición de sustituir a los trabajadores en huelga, la inexistencia de riesgo para la vida o salud de los trabajadores y la prohibición de discriminación.

2 - El principio de eficacia, por cuanto la ordenación de la carrera administrativa no se establece en exclusivo beneficio de los empleados, sino también de la administración para que ésta pueda servir eficazmente a los intereses generales, y este efecto se logra con el desempeño de los puestos administrativos por los más capacitados.

- El principio de jerarquía, que se manifiesta en la diversificación de categorías, que impiden un tratamiento unitario.

- El principio de promoción a través del trabajo, de plena aplicación en el ámbito de la función pú14 blica (Ver STC 50/86, de 23 de abril) que conlleva, en su vertiente dinán 
ni en la capacidad de organización conforme a una de ellas únicamente" (Férez Fernández, 2006: 23; Palomar Olmeda, 2007: 213-214).

En el ámbito de las retribuciones del sector público, y en contraste con el interés demostrado por el sector privado en el diseño de modelos, las entidades públicas han hecho un menor esfuerzo en su medición y registro. $Y$ ello a pesar de su indudable interés, pues la intangibilidad del capital intelectual está incluso más presente en el sector público que en las empresas privadas (Ramió, 2002).

La adopción de un modelo retributivo concreto responde a diversas variables que cada organización debe definir en función de su estrategia, estructura organizacional, cultura, valores corporativos, objetivos, misión, etc. Para Milgrom y Roberts (1992), la teoría convencional de la agencia supone una representación razonable de las relaciones de agencia que se observan en el ámbito público, por lo que la estructura conceptual que suministra esta teoría resulta de gran utilidad para analizar el funcionamiento y la gestión de las organizaciones públicas.

También es importante que cada organización se plantee cuál es el andamiaje mínimo necesario para desarrollar un sistema retributivo. En este sentido, Milkovich y Newman (1996) desarrollan cuatro líneas generales: equidad interna, equidad externa, rendimiento del empleado y modelo de gestión del sistema salarial.

Este planteamiento supone centrar la atención en el puesto como criterio básico de compensación, lo cual equivale a adoptar la valoración del puesto como fundamento de la remuneración (Fernández Ríos y Sánchez, 1997).

EI EBEP establece que la retribución del personal laboral se determinará de acuerdo con la legislación laboral, el convenio colectivo que sea aplicable y el contrato de trabajo. Existe asimismo un límite, el fijado en el artículo 21.2, que impide que se acuerden incrementos retributivos que globalmente supongan un incremento de la masa salarial superior a los límites fijados anualmente en la Ley de Presupuestos Generales del Estado.

El artículo 26 del Estatuto de los Trabajadores plantea un escenario normativo en el cual se desenvuelve o proyecta la estructura o composición del salario, definiendo como tal la "totalidad de las prestaciones económicas de los trabajadores, en dinero o especie, por la prestación profesional de los servicios laborales por cuenta ajena, ya retribuyan el trabajo efectivo cualquiera que sea la forma de remuneración, o los períodos de descanso computables como de trabajo."

Especial importancia tiene la evaluación del desempeño de los empleados que las administraciones públicas deberán establecer a través de procedimientos fundados en los principios de igualdad, objetividad y transparencia. A tal efecto, el artículo 24 del EBEP, sobre retribuciones complementarias de los empleados públicos en general, relaciona éstas con el grado de interés, iniciativa o esfuerzo y con el rendimien- 
to o resultados obtenidos. Y el artículo 20, que regula la evaluación del desempeño, incluye entre sus efectos, los relativos al cómputo de las retribuciones complementarias.

Por otra parte, hay que hacer hincapié en que la implantación de un sistema de evaluación del desempeño exige el desarrollo, en las organizaciones públicas, de importantes capacidades internas: sistemas de definición de objetivos, indicadores de rendimiento, instrumentos para valorar competencias, técnicos especializados, directivos capacitados y comprometidos, etc. Ello supone una mayor inversión en la gestión de recursos humanos y el refuerzo de las unidades especializadas dedicadas a dicha gestión (Francis y Holloway, 2002: 283-291).

En cualquier caso, y a pesar de las dificultades de medición, atribución y establecimiento de recompensas, no cabe duda que el diseño institucional de esquemas de indicadores de gestión, que permitan la evaluación de resultados, es un elemento fundamental en la implantación de mecanismos contractuales de incentivos.

\section{EL SISTEMA DE CLASIFICACION PROFESIONAL}

\section{1) Bases del modelo}

La clasificación profesional constituye una pieza clave para determinar el régimen jurídico de la relación laboral y para concretar el contenido de la prestación. Es el punto de referencia que acota el modo y forma en que debe realizarse la prestación laboral.

Es también el grado de reconocimiento de la aportación de valor que realiza con su trabajo el empleado, en dos aspectos:

- Desde el punto de vista organizativo, determina las posibilidades de flexibilidad en la organización del trabajo y de la planificación de la plantilla.

- Desde el punto de vista del trabajador, constituye el reconocimiento de su profesionalidad y establece el alcance del contenido de la prestación laboral.

Un buen modelo de clasificación profesional debe ser capaz de potenciar el desarrollo profesional y laboral de los empleados, favorecer la movilidad funcional dentro de espacios organizativos y aportar unas reglas claras y conocidas por todos que permitan estimar en todo momento las perspectivas profesionales de cada uno de los empleados.

El modelo que se contempla en este trabajo pretende dar respuesta a esos requerimientos. Ordena los puestos de trabajo de la institución atendiendo a los niveles 16 de titulación, formación y capacitación para ejercer los cometidos de los distintos ni- 
veles, facilitar la movilidad del personal y favorecer su promoción, estableciendo para ello mecanismos de carrera.

Para poder elaborar el sistema de clasificación profesional que se propone, se necesitan dos documentos. El primero es el organigrama de la institución que se presenta en el gráfico 1 y consta de 12 unidades administrativas, 6 Departamentos, 2 Subdirecciones y 2 Direcciones. En la cúpula se encuentra la Presidencia.

\section{GRÁFICO 1}

\section{Organigrama de la institución}

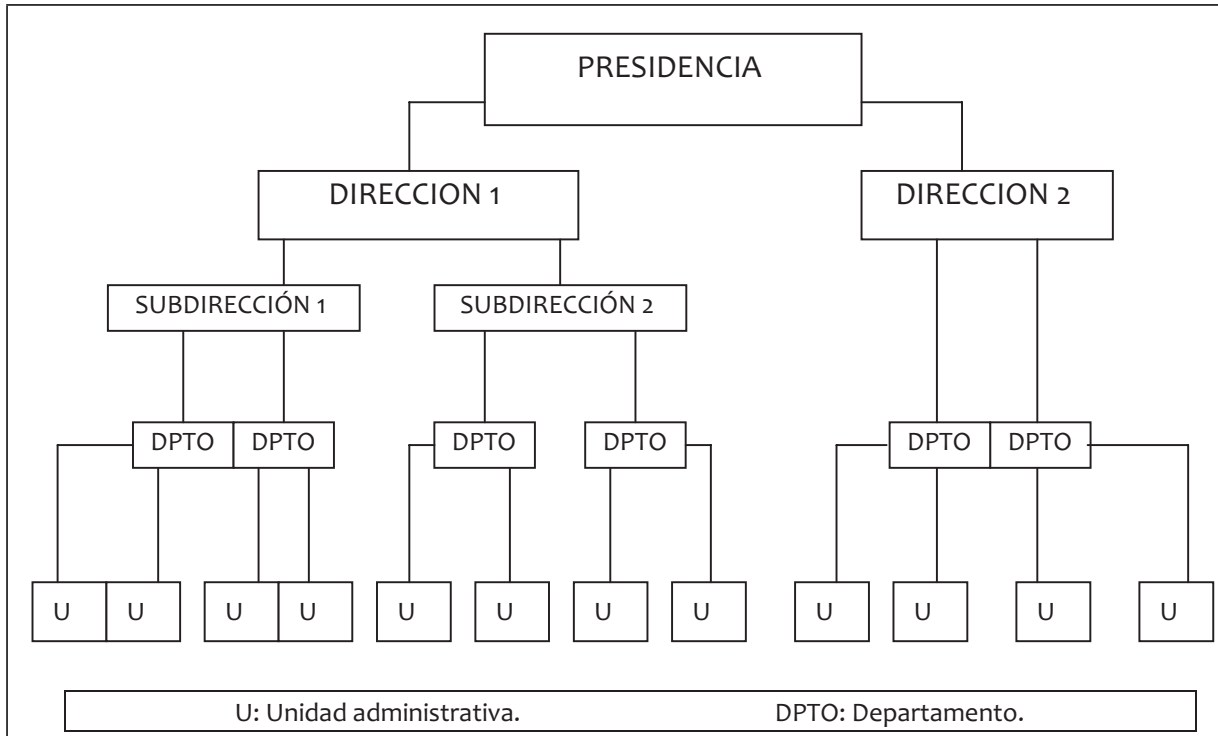

Las unidades administrativas son los elementos organizativos básicos de la estructura orgánica que comprenden puestos de trabajo o dotaciones de plantilla vinculados funcionalmente en razón de sus cometidos y, orgánicamente, por una jefatura de Departamento común. Los Departamentos pueden estar adscritos a una Dirección o Subdirección y ésta a una de las Direcciones que dependen directamente de la Presidencia.

Fuente: Elaboración propia.

El segundo es un informe elaborado en 2004 para la institución por la Consultoría Operativa de Servicios del, entonces, Ministerio de Administraciones Públicas $(\mathrm{MAP})^{3}$ en el que se analizan exhaustivamente:

3 El Sistema de Análisis Organizacional (SAO) ha sido el método elegido por el MAP para llevar a cabo un análisis de la estructura y de las funciones de los distintos departamentos y servicios administrativos y profesionales de la institución. La primera fase requiere un trabajo de gabinete en el que se recopila información institucional. En la siguiente fase, se recopila información directamente de los colectivos afectados mediante cuestionarios y entrevistas personales. Las definiciones de los términos utilizados por este método tienen un carácter operativo, es decir, únicamente son utilizables en el contexto de esta metodología: 
- Las funciones asignadas y su concordancia con las realizaciones de cada dotación.

- Las tareas más frecuentes y más complejas de cada dotación.

- Los procedimientos para llevarlas a cabo.

- Las condiciones en las que se realizan las tareas (equipos, herramientas, supervisión, coordinación y control, elementos que dificultan la ejecución, etc.)

- Conocimientos necesarios para poder ejecutar las tareas (marco de referencia).

- Aptitudes básicas para poder aprender el puesto y adaptarse a las exigencias cambiantes del mismo.

- Destrezas en las que se concreta el saber hacer del puesto de trabajo; es decir, conocimientos+aptitudes+experiencia y entrenamiento.

Partiendo de esos datos, y en orden a elaborar el cuadro de clasificación profesional, se ha seguido la siguiente secuencia metodológica:

a) Identificación de "puestos tipo" a partir del análisis de funciones contenido en el informe citado.

b) Clasificación y ordenación de los puestos tipo en grupos, niveles y categorías.

c) Codificación de los puestos tipo a partir de su clasificación y ordenación.

d) Valoración de los puestos mediante la asignación de coeficientes.

- Función: Cometido homogéneo desarrollado por una Unidad Administrativa para producir o proveer un servicio o prestación.

- Actividad: Cada una de las acciones, con inicio y final identificables, ejecutadas en una Unidad Administrativa para el desarrollo de una Función.

- Tareas Genéricas: Relación de tareas comunes en las distintas unidades administrativas por medio de las que se verifica materialmente una actividad.

A través de estos tres conceptos se deben poder determinar los objetivos de las unidades y los cometidos de cada empleado. En esta fase de actuaciones se pretenden delimitar las actividades que se realizan por cada puesto de trabajo diferenciado.

Para realizar la definición de las actividades, se elaboraron dos listados: el primero describe funciones y actividades específicas de las distintas unidades que componen la estructura administrativa de la institución y el segundo se refiere a actividades comunes que se realizan en todos los departamentos y 18 servicios, y debe servir para adecuar los niveles jerárquicos a la asignación del grado de responsabilidad 


\section{a) Identificación de puestos tipo}

El puesto tipo agrupa unitariamente el contenido concreto de la prestación laboral y hace referencia a las tareas que se desarrollan en el puesto de trabajo. El análisis de funciones, en este caso, da lugar a quince puestos-tipo:

1. Director. El personal que ocupa este puesto tipo elabora las políticas y directrices de la institución. Tiene alto nivel de responsabilidad en la dirección, planificación, organización, gestión y control, y alto grado de exigencia en los factores de iniciativa, autonomía y responsabilidad.

2. Subdirector. Es el responsable directo de la correcta aplicación de las políticas y directrices elaboradas a nivel de Director. Organiza los factores, se encarga de que se ejecuten las decisiones, controla las posibles desviaciones entre los resultados obtenidos y los deseados. Su actividad implica mando sobre equipos de personas y responsabilidad alta en la gestión de recursos humanos, económicos o financieros.

3. Jefe de Departamento Coordinador. En este puesto se desarrollan funciones predirectivas propias de la Dirección o Subdirección a la que esté adscrito, y con muy alto nivel de especialización. Su desempeño se traduce en la realización de cometidos relacionados con la planificación, evaluación y previsión u otros de análoga naturaleza, organizando y controlando los procesos de trabajo a realizar. Coordina al personal que ocupa puestos tipo 4 en las Subdirecciones y Direcciones.

4. Jefe de Departamento. En este puesto se desarrollan funciones técnicas superiores de gestión con alto nivel de responsabilidad y especialización. Su desempeño se traduce en la realización de cometidos relacionados con la investigación, estudio y análisis u otros de análoga naturaleza, organizando los procesos de trabajo a realizar y a los trabajadores que los han de llevar a cabo.

5. Asesor de Departamento. En este puesto se desarrollan funciones técnicas superiores de asesoría en la gestión de los procedimientos para llevar a la práctica los planes generales de la institución, que corresponden al Departamento al que se encuentra adscrito. Se trata de personal con alto nivel de especialización en las materias propias de su actividad profesional.

6. Jefe de unidad. El personal que ocupa este puesto es responsable del correcto funcionamiento de la unidad y de la adecuada ejecución de las tareas asignadas a la misma. En este puesto se desarrollan funciones técnicas superiores y de gestión, con alto nivel de especialización; organiza los procesos de trabajo a realizar y a los trabajadores que los han de llevar a cabo, encargándose de su motivación, integración y formación. 
7. Coordinador de Unidad. En este puesto se desarrollan funciones técnicas superiores con alto nivel de especialización. Coordina al personal que ocupa puestos tipo 8, 9 y 10 contando, para el desempeño de sus tareas, con cierto grado de autonomía para proceder a la resolución de problemas técnicos o prácticos propios de su campo de actuación y para la organización y control de los procesos de trabajo.

8. Asesor de Unidad. En este puesto se desarrollan funciones técnicas superiores con alto nivel de especialización, bajo instrucciones concretas y directas del personal que ocupa puestos tipo 7. Realiza cometidos relacionados con la investigación, estudio, análisis, asesoramiento, u otros de análoga naturaleza, sin que estas actividades conlleven organización y control de los procesos de trabajo.

9. Titulado Superior. En este puesto se desarrollan funciones técnicas superiores de apoyo para la elaboración de los estudios, informes y otros documentos técnicos cuya realización corresponda a la unidad a la que esté adscrito. También realiza funciones de gestión con medio nivel de especialización y de responsabilidad pero con diferenciación de tareas respecto al puesto tipo 8.

10. Secretaria del Presidente. En este puesto se realizan trabajos de ejecución autónoma que exigen habitualmente iniciativa, disponibilidad y alta especialización en funciones administrativas propias de los gabinetes de órganos unipersonales. Los trabajadores que ocupan estos puestos están sujetos al seguimiento de instrucciones concretas para el desempeño de sus funciones.

11. Secretaria de Director. En este puesto se desarrollan tareas de administración y gestión con dependencia directa de los Directores, y consisten en operaciones administrativas de ejecución autónoma con cierta autonomía e iniciativa, comportando, bajo supervisión, la responsabilidad de las mismas.

12. Administrativo. En este puesto se desarrollan tareas de administración y de apoyo, o complementarias, en las Direcciones de la institución. Se realizan trabajos de ejecución autónoma que exijan habitualmente iniciativa, comportando, bajo supervisión, la responsabilidad de las mismas. Las tareas consisten en operaciones administrativas, de apoyo o complementarias bajo seguimiento de instrucciones concretas.

13. Auxiliar de ofimática. En este puesto se desarrollan funciones de administración y apoyo ofimático en las Direcciones de la institución. Los trabajadores que ocupan estos puestos están sujetos al seguimiento de instrucciones detalladas para el desempeño de sus tareas que consisten en operaciones instrumentales básicas, de apoyo administrativo o complementarias.

14. Auxiliar de Servicios Generales. En este puesto se realizan tareas de ejecución autónoma con cierto grado de autonomía, que consisten en operacio- 
nes instrumentales básicas de apoyo a la gestión administrativa en las unidades administrativas.

15. Ordenanza. En este puesto se realizan trabajos de ejecución autónoma que no exigen iniciativa. Los trabajadores están sujetos al seguimiento de instrucciones detalladas para el desempeño de sus tareas que consisten en operaciones instrumentales básicas, simples, repetitivas, mecánicas o automáticas, de apoyo o complementarias.

\section{b) Clasificación de puestos en grupos, niveles y categorías}

Los quince puestos tipo se ordenan para obtener el cuadro de clasificación profesional que se muestra en el cuadro 1, a partir de los siguientes indicadores:

- Los requisitos de titulación: Con este criterio los puestos quedan clasificados en cuatro grupos.

- El tipo y complejidad del sistema de acceso, y determinados factores genéricos asociados a las funciones de los puestos tipo pertenecientes a cada grupo, dan lugar a seis niveles.

- El tipo de funciones técnicas, ejecutivas, directivas, auxiliares y de apoyo, y otros factores específicos asociadas a cada nivel, dan lugar a diez categorías.

\section{CUADRO 1}

Clasificación de puestos

\begin{tabular}{|c|c|c|c|c|}
\hline Puesto tipo Denominación & $\begin{array}{c}\text { Puesto } \\
\text { tipo }\end{array}$ & Categoría & Nivel & Grupo \\
\hline Director & 1 & 1 & \multirow{4}{*}{1} & \multirow{9}{*}{ I } \\
\hline Subdirector & 2 & 2 & & \\
\hline Jefe de Departamento Coordinador & 3 & \multirow{2}{*}{3} & & \\
\hline Jefe de Departamento & 4 & & & \\
\hline Asesor de Departamento & 5 & 4 & \multirow{4}{*}{2} & \\
\hline Jefe de Unidad & 6 & \multirow{2}{*}{5} & & \\
\hline Coordinador de Unidad & 7 & & & \\
\hline Asesor de Unidad & 8 & 6 & & \\
\hline Titulado Superior & 9 & 7 & 3 & \\
\hline Secretario/a de Presidente & 10 & \multirow{3}{*}{8} & \multirow{3}{*}{4} & \multirow{3}{*}{ II } \\
\hline Secretario/a de Director/a & 11 & & & \\
\hline Administrativo & 12 & & & \\
\hline Auxiliar de Ofimática & 13 & \multirow{2}{*}{9} & \multirow{2}{*}{5} & \multirow{2}{*}{ III } \\
\hline Auxiliar de Servicios Generales y Administración & 14 & & & \\
\hline Ordenanza & 15 & 10 & 6 & IV \\
\hline
\end{tabular}

Fuente: Elaboración propia. 
El Grupo es el componente del sistema de clasificación asociado a la titulación académica según la siguiente distribución:

- Grupo I. Título de Doctor, Licenciado, Grado, Diplomado y equivalentes. Están en este grupo los puestos tipo 1 a 9.

- Grupo II. Título de Bachillerato, Formación profesional de Técnico Superior y equivalentes. Están en este grupo los puestos tipo 10 a 12.

- Grupo III. Título de Bachillerato, Formación profesional de Técnico y equivalentes. Están en este grupo los puestos tipo 13 y 14.

- Grupo IV. Título de graduado en educación secundaria obligatoria y equivalentes. Está en este grupo el puesto tipo 15.

El nivel es el componente del sistema de clasificación asociado a los siguientes factores genéricos:

a) Complejidad del sistema de acceso: Se refiere al grado de dificultad de los procesos de selección de personal.

b) Conocimientos: Conjunto de habilidades técnicas, experiencias y habilidades requeridas para un normal desempeño de las funciones.

c) Responsabilidad: Factor para cuya valoración se tienen en cuenta el grado de autonomía de acción del trabajador, el nivel de influencia sobre los resultados, la relevancia de la gestión sobre recursos humanos, técnicos y productivos, y la asunción del riesgo por las decisiones tomadas y sus consecuencias.

d) Iniciativa: Factor para cuya valoración se tiene en cuenta el grado de dependencia jerárquica en el desempeño de funciones. Puede desarrollarse en dos ámbitos diferenciados: el requerido para afrontar conceptualmente la identificación, definición y búsqueda de soluciones a los problemas, y el requerido para adoptar decisiones y asumir sus consecuencias.

e) Mando: Factor para cuya valoración se tiene en cuenta el grado de supervisión y ordenación de las funciones y tareas, la capacidad de interrelación, las características del colectivo y el número de personas sobre las que se ejerce el mando. En su valoración deben concurrir dos tipos de capacidades: las de integración y armonización de funciones, recursos y objetivos diferentes, incidiendo de forma directa en los resultados del equipo; y las de consecución de resultados a través de otras personas, a las que se debe orientar, motivar, contribuir a desarrollar, influir y supervisar.

En el cuadro 2 se muestra la clasificación de puestos tipo en niveles según el 22 grado de exigencia en los factores señalados. 


\section{CUADRO 2}

Grados de exigencia de los factores asociados al nivel

\begin{tabular}{|c|l|l|l|l|l|l|}
\hline Nivel & $\begin{array}{c}\text { Complejidad de } \\
\text { acceso }\end{array}$ & Conocimientos & Responsabilidad & Iniciativa & Mando & $\begin{array}{c}\text { Puestos } \\
\text { tipo }\end{array}$ \\
\hline $\mathbf{1}$ & Muy alto & Muy alto & Muy alto & Muy alto & Muy alto & 1 a 4 \\
\hline $\mathbf{2}$ & Muy alto & Muy alto & Alto & Alto & Alto & 5 a 8 \\
\hline $\mathbf{3}$ & Alto & Alto & Medio & Medio & Medio & 9 \\
\hline $\mathbf{4}$ & Medio & Medio & Medio & Bajo & Bajo & 10 a 12 \\
\hline $\mathbf{5}$ & Medio & Medio & Bajo & Bajo & Ninguno & 13 y 14 \\
\hline $\mathbf{6}$ & Bajo & Bajo & Bajo & Ninguno & Ninguno & 15 \\
\hline
\end{tabular}

Fuente: Elaboración propia.

La categoría profesional se define por su pertenencia a un grupo y nivel, recoge de manera no exhaustiva las actividades propias de los puestos y agrupa unitariamente el contenido general de la prestación laboral y las aptitudes profesionales que se corresponden con las mismas.

El contenido general de la prestación hace referencia a las actividades profesionales que se desarrollan en la institución, en sus respectivas unidades organizativas y funcionales. La aptitud profesional es el resultado de la ponderación de una serie de factores específicos, en relación con los factores genéricos de nivel ya expuestos. Dichos factores específicos son los siguientes:

- Formación básica

- Formación específica (técnica/administrativa)

- Formación específica (institucional)

- Actualización

- Requerimientos intelectivos

- Especialización profesional ${ }^{4}$

- Experiencia previa

- Habilidades operativas

- Habilidades sociales

— Impacto sobre las personas

- Impacto sobre la institución

- Planificación

- Toma de decisiones

- Organización a nivel de unidad administrativa

- Organización a nivel institucional

- Supervisión

4 Factor que se diferencia de la formación específica en que se refiere a las aptitudes, conocimientos y capacidades para la realización de funciones en un área específica dentro de las incluidas en las actividades de la institución. 
- Autonomía

- Representación

- Coordinación

- Seguimiento de actividades a nivel de unidad administrativa

- Seguimiento de actividades a nivel institucional

- Control de actividades a nivel de unidad administrativa

- Control de actividades a nivel institucional

- Tramitación de expedientes

Del análisis realizado resultan diez categorías: tipo 1).

1: Corresponde al personal directivo definido en el artículo 13 del EBEP (puesto

2: Personal directivo que apoya al de la categoría 1 en la planificación de objetivos generales de la institución (puesto tipo 2).

3: Personal predirectivo cuyas funciones consisten en la realización de una actividad profesional de carácter específico y con responsabilidad directa en la consecución de los objetivos de la institución (puestos tipo 3 y 4).

4: Personal técnico y de gestión de alta cualificación y especialización profesional que tiene responsabilidad directa en la consecución de los objetivos del Departamento (puesto tipo 5).

5: Personal de gestión de alta cualificación profesional cuyas funciones consisten en la realización de actividades complejas y responsabilidad directa en la consecución de los objetivos de la Unidad (puesto tipo 2).

6: Personal técnico de alta cualificación cuyas funciones consisten en la realización de actividades de contenido intelectual y alto grado de exigencia en el factor de autonomía (puesto tipo 8).

7: Personal que realiza funciones consistentes en la realización de actividades homogéneas de ejecución autónoma que exijan iniciativa comportando la responsabilidad de las mismas (puesto tipo 9).

8: Personal que realiza trabajos de ejecución autónoma con sujeción a procedimientos establecidos y al seguimiento de instrucciones concretas (puestos tipo 10 a 12).

9: Personal que realiza trabajos de ejecución autónoma bajo supervisión, que consisten en operaciones ofimáticas, administrativas, de apoyo y complementarias (puesto tipo 13 y 14).

24 (puesto tipo 15).

10: Personal que realiza trabajos básicos de carácter instrumental y genérico 


\section{c) Códigos de clasificación}

La identificación de cada puesto tipo se realiza mediante códigos numéricos o por coeficientes alfanuméricos.

En el primer caso, el primer elemento identifica el grupo, el segundo el nivel, el tercero la categoría y el cuarto el puesto tipo. A efectos de carrera, como se verá más adelante, se añade un quinto componente ${ }^{5}$. En el segundo caso, se sigue el mismo criterio de identificación, solo que en lugar de dígitos numéricos se utilizan los coeficientes $\alpha, \beta, \gamma$ y $\delta$ para el nivel, la categoría, el puesto tipo y la carrera, respectivamente (cuadro 3 ).

\section{CUADRO 3}

Cuadro de clasificación general mediante códigos

\begin{tabular}{|c|c|c|c|}
\hline $\begin{array}{l}\text { Puesto } \\
\text { Tipo }\end{array}$ & Denominación & $\begin{array}{l}\text { Por números } \\
\text { dígitos }\end{array}$ & $\begin{array}{c}\text { Por } \\
\text { coeficientes }\end{array}$ \\
\hline 1 & Director & I.1.1.1.1 & $\alpha_{1} \beta_{1} \gamma_{1} \delta_{i}$ \\
\hline 2 & Subdirector & I.1.2.2.2 & $\alpha_{1} B_{2} \gamma_{2} \delta_{2}$ \\
\hline 3 & Jefe de Departamento Coordinador & I.1.3.3.3 & $\alpha_{1} \theta_{3} \gamma_{3} \delta_{3}$ \\
\hline 4 & Jefe de Departamento & I.1.3.4.3 & $\alpha_{1} \beta_{3} \gamma_{4} \delta_{3}$ \\
\hline 5 & Asesor de Departamento & I.2.4.5.4 & $\alpha_{2} B_{4} \gamma_{5} \delta_{4}$ \\
\hline 6 & Jefe de Unidad & $1.2 .5 \cdot 6.5$ & $\alpha_{2} b_{5} \gamma_{6} \delta_{5}$ \\
\hline 7 & Asesor-Coordinador de Unidad & $1.2 \cdot 5 \cdot 7 \cdot 5$ & $\alpha_{2} b_{6} \gamma_{7} \delta_{5}$ \\
\hline 8 & Asesor de Unidad & I.2.6.8.6 & $\alpha_{2} B_{6} \gamma_{8} \delta_{6}$ \\
\hline 9 & Titulado Superior & $1.3 \cdot 7 \cdot 9 \cdot 7$ & $\alpha_{3} \beta_{7} \gamma_{9} \delta_{7}$ \\
\hline 10 & Secretario/a de Órganos unipersonales & II.4.8.10.8 & $\alpha_{4} \beta_{8} \gamma_{10} \delta_{8}$ \\
\hline 11 & Secretario/a de Director/a & II.4.8.11.8 & $\alpha_{4} \beta_{8} \gamma_{11} \delta_{8^{\circ}}$ \\
\hline 12 & Administrativo & II.4.8.12.8 & $\alpha_{4} \beta_{8} \gamma_{12} \delta_{g^{\circ}}$ \\
\hline 13 & Auxiliar de Ofimática & III.5.9.13.9 & $\alpha_{5} \beta_{9} \gamma_{13} \delta_{9}$ \\
\hline 14 & Auxiliar de Servicios Generales y Administración & III.5.9.14.9 & $\alpha_{5} b_{9} \gamma_{14} \delta_{9}$ \\
\hline 15 & Ordenanza & IV.6.10.15.10 & $\alpha_{6} \beta_{10} \gamma_{15} \delta_{10}$ \\
\hline
\end{tabular}

Fuente: Elaboración propia.

\section{d) Valoración de puestos}

La valoración es el procedimiento mediante el cual se pretende determinar el valor relativo de los distintos puestos que componen la organización. En nuestro

5 Así, por ejemplo, el código I.2.6.8.6 corresponde al puesto perteneciente al grupo I, nivel 2, categoría 6, puesto tipo 8 y componente de carrera 6, cuya denominación es la de Asesor de Unidad. 
caso, el primer paso consiste en cuantificar los coeficientes de clasificación y el segundo en utilizar dichos coeficientes para articular el sistema retributivo, tarea que se realizará más adelante.

La cuantificación de los coeficientes de clasificación se realiza en función del resultado de la valoración de los factores técnicos genéricos y específicos que ya se han visto. El cuadro 4 muestra los valores de los coeficientes cuyo método de cálculo se expone a continuación.

\section{CUADRO 4}

Valores de los coeficientes de clasificación

\begin{tabular}{|c|c|c|c|c|}
\hline & $a_{i}$ & $\beta_{\mathrm{i}}$ & $\gamma_{p}$ & $\delta_{i}$ \\
\hline \multirow{9}{*}{ I } & \multirow{4}{*}{$a_{1}=7,973$} & $\beta_{1}=0,60$ & $\gamma_{1}=0,70$ & $\delta_{1}=0,025$ \\
\hline & & $\beta_{2}=0,55$ & $\gamma_{2}=0,60$ & $\delta_{2}=0,028$ \\
\hline & & \multirow{2}{*}{$\beta_{3}=0,50$} & $\gamma_{3}=0,40$ & $\delta_{3}=0,030$ \\
\hline & & & $\gamma_{4}=0,20$ & $\delta_{3}=0,030$ \\
\hline & \multirow{4}{*}{$a_{2}=6,525$} & $\beta_{4}=0,50$ & $\gamma_{5}=0,50$ & $\delta_{4}=0,030$ \\
\hline & & \multirow{2}{*}{$\beta_{5}=0,45$} & $\gamma_{6}=0,40$ & $\delta_{5}=0,034$ \\
\hline & & & $\gamma_{7}=0,25$ & $\delta_{5}=0,034$ \\
\hline & & $\beta_{6}=0,40$ & $\gamma_{8}=0,10$ & $\delta_{6}=0,038$ \\
\hline & $\alpha_{3}=5,078$ & $\beta_{7}=0,45$ & $\gamma_{9}=0,40$ & $\delta_{7}=0,034$ \\
\hline \multirow{3}{*}{ II } & \multirow{3}{*}{$a_{4}=3,630$} & \multirow{3}{*}{$\beta_{8=0,50}$} & $\gamma_{10}=0,60$ & $\delta_{8}=0,032$ \\
\hline & & & $\gamma_{11}=0,30$ & $\delta_{8}=0,032$ \\
\hline & & & $\gamma_{12}=0,10$ & $\delta_{8}=0,032$ \\
\hline \multirow{2}{*}{ III } & \multirow{2}{*}{$a_{5}=2,183$} & \multirow{2}{*}{$\beta_{9}=0,50$} & $\gamma_{13}=0,40$ & $\delta_{9}=0,034$ \\
\hline & & & $\gamma_{14}=0,10$ & $\delta_{9}=0,034$ \\
\hline IV & $a_{6}=1$ & $\beta_{10}=0,50$ & $\gamma_{15}=0,30$ & $\delta_{10}=0,036$ \\
\hline
\end{tabular}

Fuente: Elaboración propia.

De la información contenida en el documento de trabajo en el que se describen las funciones de los puestos y del cuadro que refleja el grado de exigencia de los factores genéricos (cuadro 2), resulta que las funciones asociadas al nivel 5 tienen una valoración superior en un $20 \%$ al nivel 6 ; es decir, la razón de progresión entre el nivel 6 y el 5 es $r_{1}=0,20$.

Las funciones asociadas al nivel 4 tienen una valoración superior en un $25 \%$ al nivel 5; es decir, la razón de progresión entre el nivel 5 y el 4 es $r_{2}=0,25$.

A partir de éste, todas las razones de progresión, hasta el primer nivel, crecen 26 también en una proporción del $25 \%$ entre niveles consecutivos $\left(r_{2}=0,25\right)$. 
De esta forma, el componente de nivel de la valoración de funciones $\left(V n_{i}\right)$ responde a la siguiente secuencia:

Primer término ${ }^{6}: \mathrm{Vn}_{6}=(1+\mathrm{x})^{\alpha^{6}}$

Segundo término: $V n_{5}=(1+x)^{a 5}=(1+x)^{a 6 \cdot}\left(1+r_{1}\right) \cdot\left(1+r_{2}\right)^{\circ}$

Tercer término: $V n_{4}=(1+x)^{a 4}=(1+x)^{a b} \cdot\left(1+r_{1}\right) \cdot\left(1+r_{2}\right)^{1}$

Cuarto término: $V n_{3}=(1+x)^{a_{3}}=(1+x)^{a} \cdot\left(1+r_{1}\right) \cdot\left(1+r_{2}\right)^{2}$

Quinto término: $V n_{2}=(1+x)^{a^{2}}=(1+x)^{a 6} \cdot\left(1+r_{1}\right) \cdot\left(1+r_{2}\right)^{3}$

Sexto término: $V n_{1}=(1+x)^{a^{1}}=(1+x)^{a 6} \cdot\left(1+r_{1}\right) \cdot\left(1+r_{2}\right)^{4}$

En esta secuencia, el término general es:

$$
V n_{i}=(1+x)^{a i}=(1+x)^{a 6} \cdot\left(1+r_{1}\right) \cdot\left(1+r_{2}\right)^{5-i}
$$

Ecuación que, expresada en términos logarítmicos, permite obtener los coeficientes $\alpha_{\mathrm{i}}$

$$
a_{i}=a_{6}+\log \left(1+r_{1}\right) \cdot\left(1+r_{2}\right)^{5-i} / \log (1+x) \text {, donde } a_{6}=1 ; r_{1}=0,20 ; r_{2}=0,25 \text { y x = 1/6 }
$$

Así se obtienen los valores que se reflejan en la columna segunda del cuadro $4^{7}$.

- Coeficiente 6 . Corresponde a la categoría profesional, representa el peso de esta en cada nivel y su valor se obtiene utilizando la información contenida en el documento de trabajo, mediante un procedimiento de ponderación adecuada de los factores genéricos (complejidad en el acceso, conocimientos, responsabilidad, iniciativa y mando) y específicos (formación, actualización, requerimientos intelectivos, experiencia previa, habilidades, etc.).

La fórmula utilizada es: $\beta_{\mathrm{j}}=\Sigma\left[\mathrm{g}_{\mathrm{FG}}\left(\Sigma e_{\mathrm{FE}}\right)\right] / \alpha_{\mathrm{i}}$; donde $\mathrm{g}_{\mathrm{FG}}$ son los pesos relativos de los factores genéricos y $e_{F E}$ los de los específicos, y sus valores se reflejan en la columna tercera del cuadro 4.

En concreto, el método consiste en utilizar, para cada categoría, una ficha que es una tabla de doble entrada en donde los factores genéricos (conocimientos, iniciativa, etc.), con sus ponderaciones de nivel, figuran en la primera fila. Los factores específicos se ordenan en la primera columna y se rellenan las cuadrículas con los pesos relativos de cada factor específico en cada factor genérico como se muestra en los cuadros 5 a 14.

$6(1+x)$ es la base del término exponencial que indica la altura de comienzo de la curva de la progresión geométrica. Se ha adoptado el valor de $x=1 / 6$ (ya que hay seis niveles), aunque nada impide adoptar cualquier otro parámetro como base y que tenga un valor razonable para su manejo matemático. Por su parte, ${ }^{a}{ }^{6}$ adopta el valor de la unidad por considerarlo más cómodo como valor de referencia para la operatividad matemática. Por tanto, la valoración para el primer término (nivel 6) es de 7/6.

$7 \quad a_{5}=1+\log (1+0,2) \cdot(1+0,25)^{5-5} / \log (1+1 / 6)=2,183$

$a_{4}=1+\log (1+0,2) \cdot(1+0,25)^{5-4} / \log (1+1 / 6)=3,630$

$a_{3}=1+\log (1+0,2) \cdot(1+0,25)^{5-3} / \log (1+1 / 6)=5,078$

$a_{2}=1+\log (1+0,2) \cdot(1+0,25)^{5-2} / \log (1+1 / 6)=6,525$

$a_{1}=1+\log (1+0,2) \cdot(1+0,25)^{5-1} / \log (1+1 / 6)=7,973$ 
CUADRO 5

Determinación del coeficiente de la categoría $1\left(B_{1}\right)$

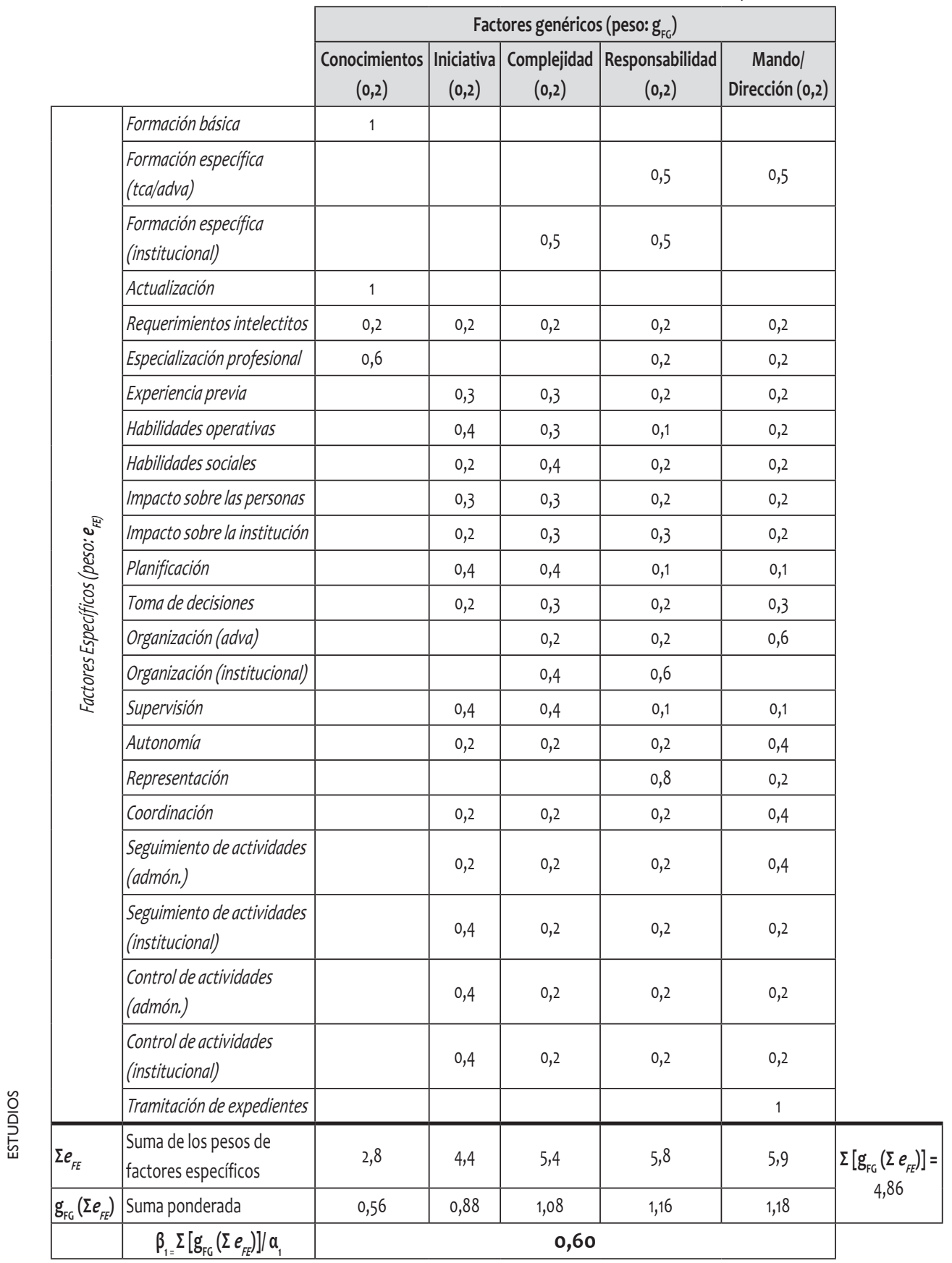

28 Fuente: Elaboración propia. 


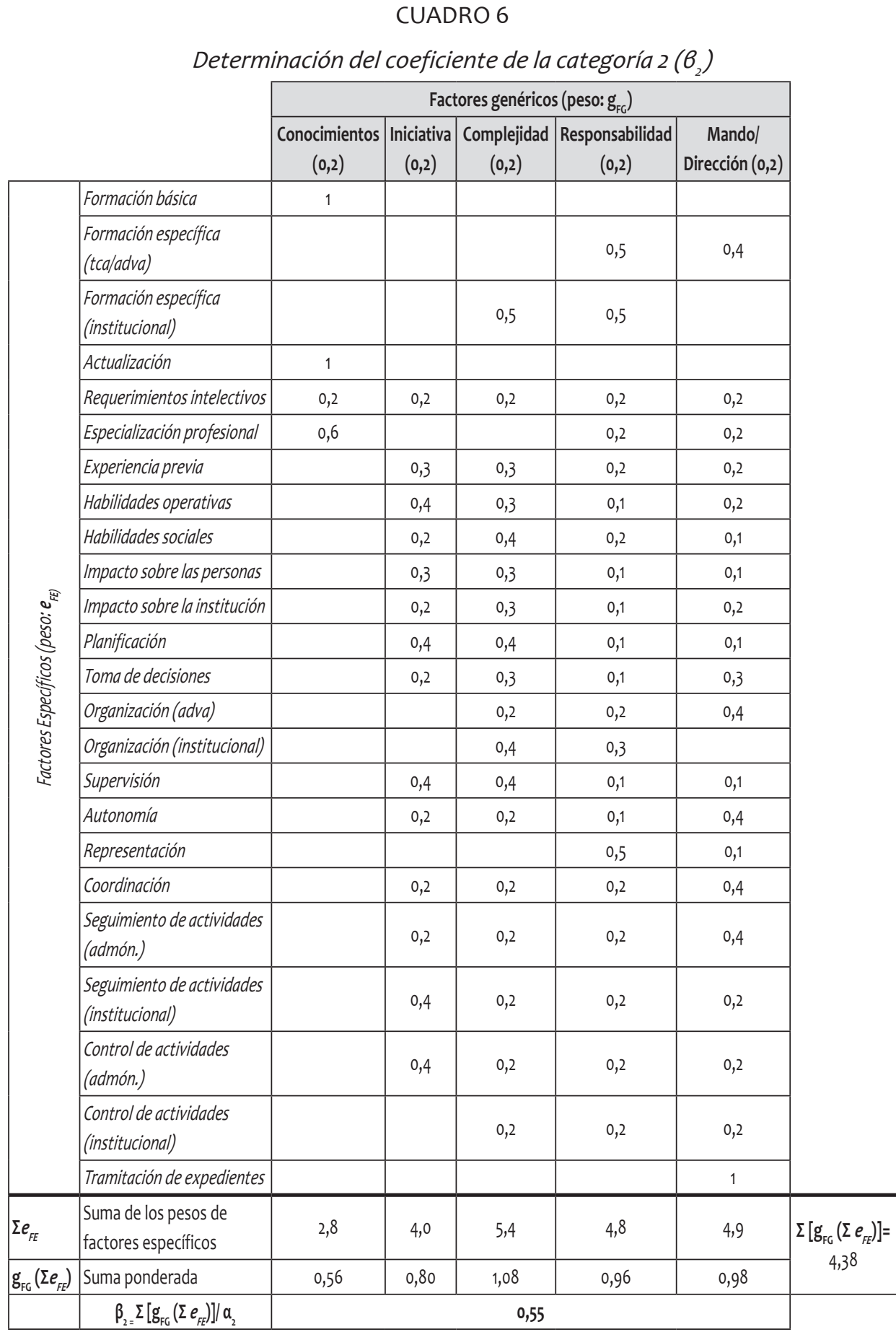


CUADRO 7

Determinación del coeficiente de la categoría $3\left(B_{3}\right)$

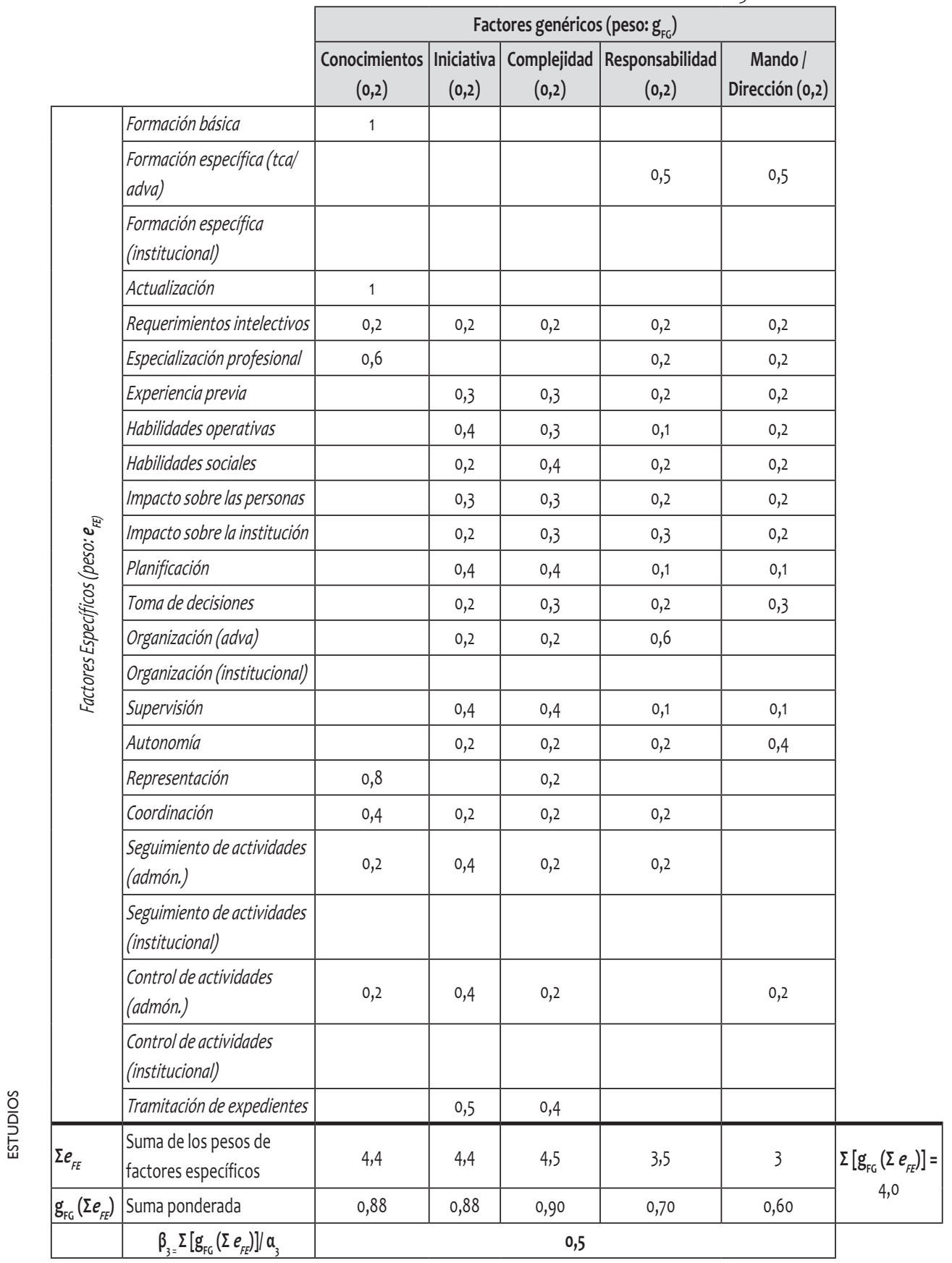

30 Fuente: Elaboración propia. 


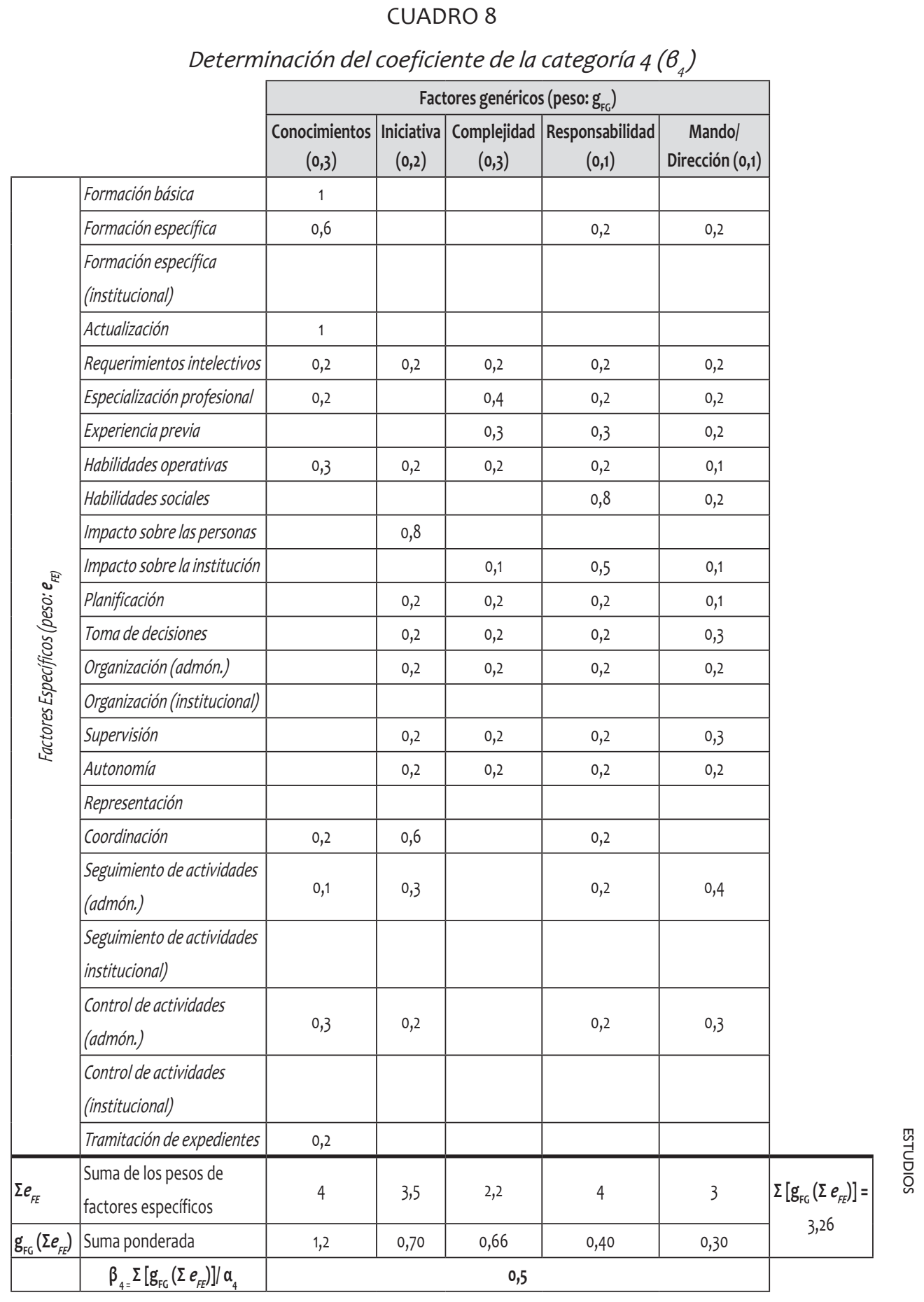

Fuente: Elaboración propia. 
CUADRO 9

Determinación del coeficiente de la categoría $5\left(B_{5}\right)$

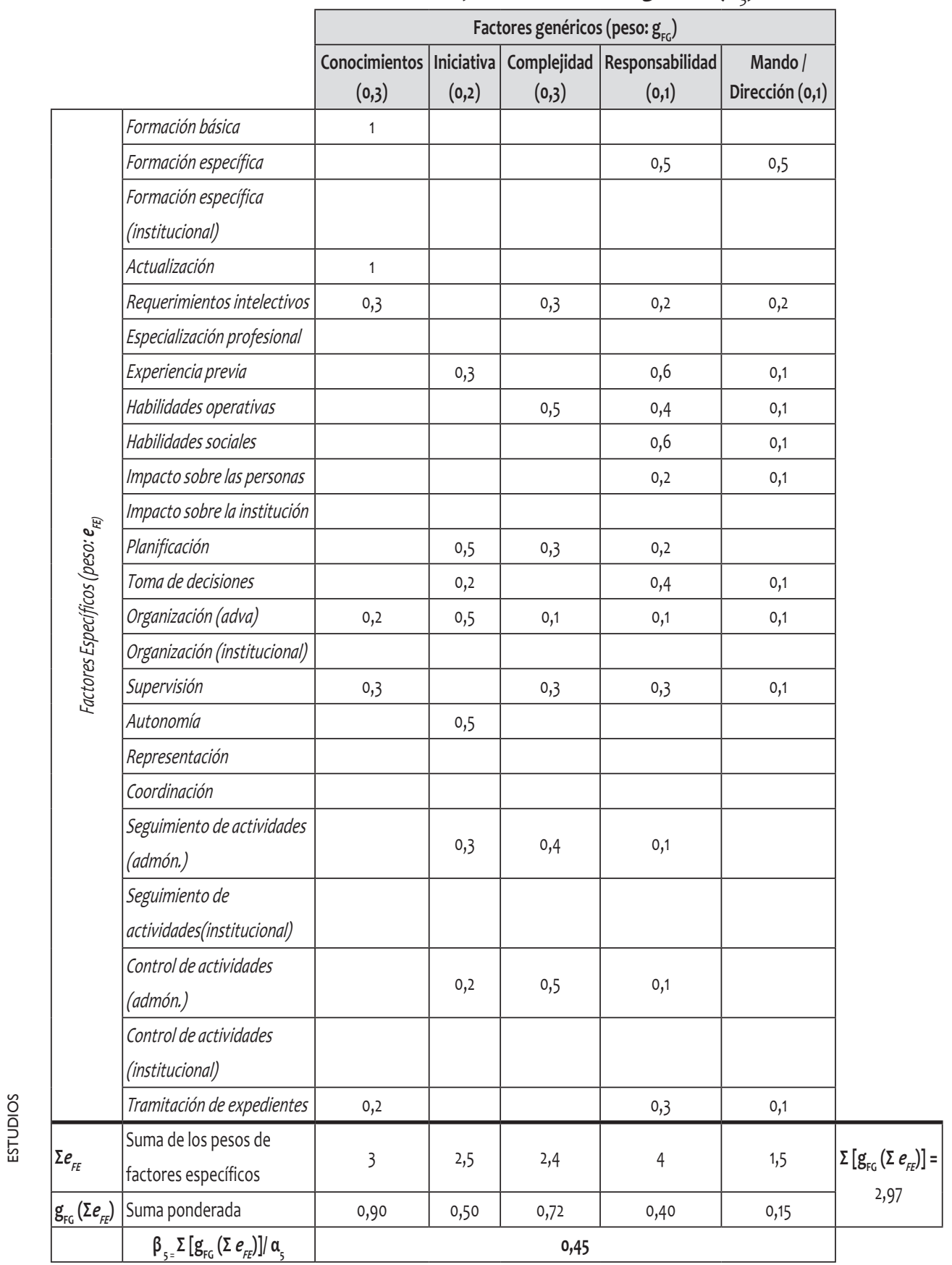

32 Fuente: Elaboración propia. 


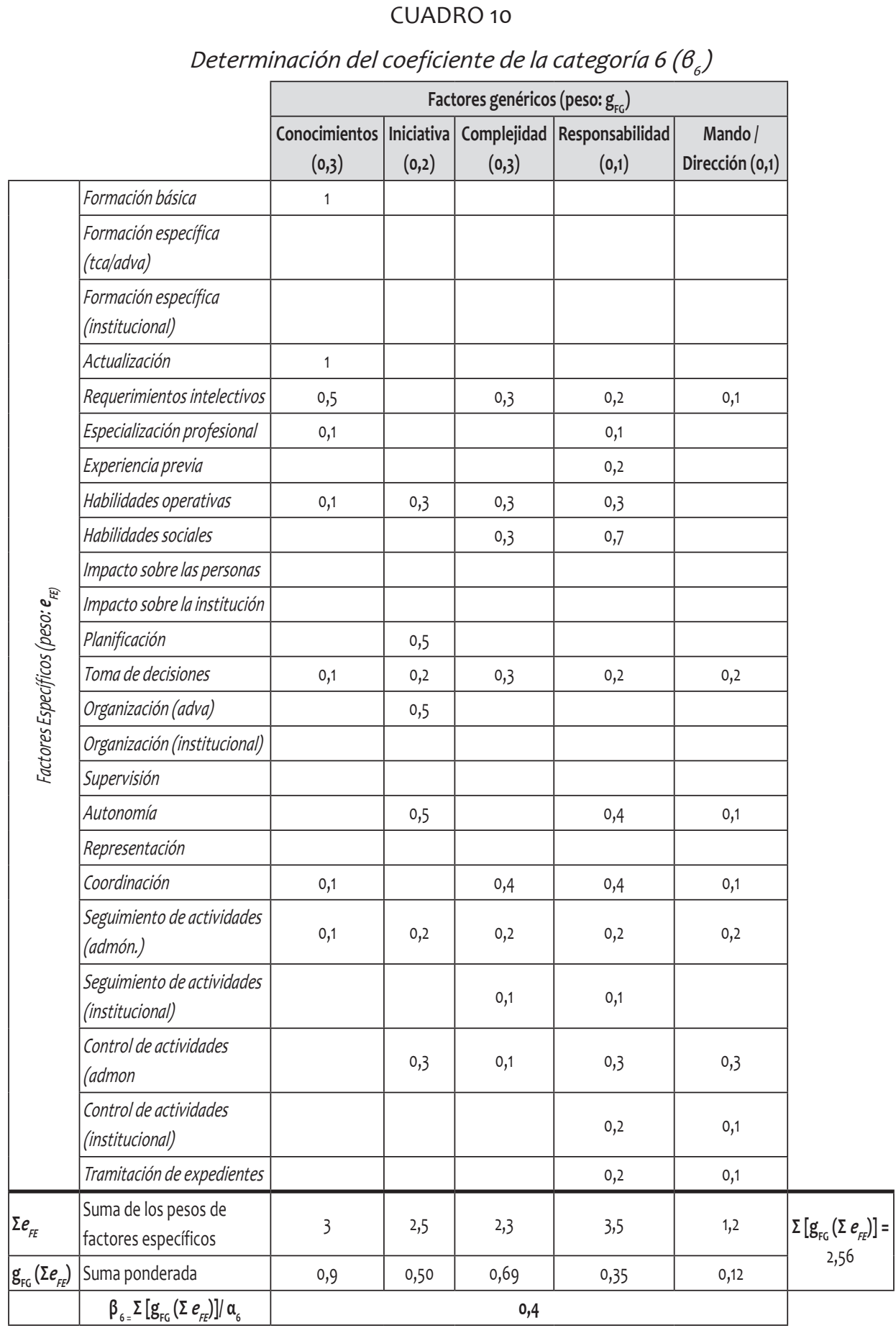


CUADRO 11

Determinación del coeficiente de la categoría $7\left(B_{7}\right)$

\begin{tabular}{|c|c|c|c|c|c|c|c|}
\hline & & \multirow{2}{*}{\multicolumn{5}{|c|}{ Factores genéricos (peso: $\mathrm{g}_{\mathrm{FG}}$ ) }} & \\
\hline & & & & & & & \\
\hline & & $\begin{array}{c}\text { Conocimientos } \\
(0,4)\end{array}$ & \begin{tabular}{|c|} 
Iniciativa \\
$(0,3)$
\end{tabular} & $\begin{array}{c}\text { Complejidad } \\
(0,2)\end{array}$ & $\begin{array}{c}\text { Responsabilidad } \\
(0,1)\end{array}$ & $\begin{array}{c}\text { Mando / } \\
\text { Dirección (0) }\end{array}$ & \\
\hline \multirow{24}{*}{ 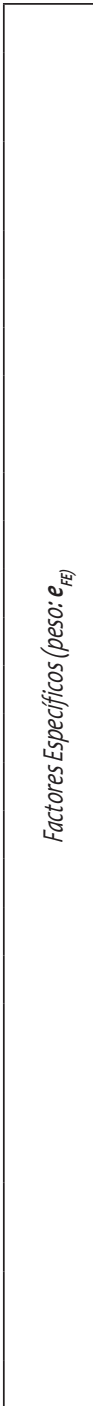 } & Formación básica & 1 & & & & & \\
\hline & $\begin{array}{l}\text { Formación especifica } \\
\text { (tca/adva) }\end{array}$ & & & & & & \\
\hline & $\begin{array}{l}\text { Formación específica } \\
\text { (institucional) }\end{array}$ & & & & & & \\
\hline & Actualización & 1 & & & & & \\
\hline & Requerimientos intelectivos & 0,8 & 0,2 & & & & \\
\hline & \begin{tabular}{|l} 
Especialización profesional \\
\end{tabular} & & & & & & \\
\hline & Experiencia previa & & & & & & \\
\hline & Habilidades operativas & & 0,4 & 0,4 & 0,2 & & \\
\hline & Habilidades sociales & & 0,4 & 0,4 & 0,2 & & \\
\hline & Impacto sobre las personas & & & & & & \\
\hline & Impacto sobre la institución & & & & & & \\
\hline & Planificación & & & & & & \\
\hline & Toma de decisiones & & & & & & \\
\hline & Organización (adva) & & 0,5 & 0,4 & 0,1 & & \\
\hline & Organización (institucional) & & & & & & \\
\hline & Supervisión & & & 0,2 & 0,3 & & \\
\hline & Autonomía & & 0,2 & 0,2 & 0,3 & & \\
\hline & Representación & & & & & & \\
\hline & Coordinación & & & & & & \\
\hline & $\begin{array}{l}\text { Seguimiento de actividades } \\
\text { (admón.) }\end{array}$ & & & & & & \\
\hline & \begin{tabular}{|l} 
Seguimiento de actividades \\
(institucional)
\end{tabular} & & & & & & \\
\hline & $\begin{array}{l}\text { Control de actividades } \\
\text { (admón.) }\end{array}$ & & 0,3 & 0,3 & 0,1 & & \\
\hline & $\begin{array}{l}\text { Control de actividades } \\
\text { (institucional) }\end{array}$ & & & & & & \\
\hline & Tramitación de expedientes & & & & & & \\
\hline$\Sigma e_{F E}$ & $\begin{array}{l}\text { Suma de los pesos de } \\
\text { factores específicos }\end{array}$ & 2,8 & 2,0 & 2,0 & 1,3 & & $\Sigma\left[\mathrm{g}_{\mathrm{FG}}\left(\Sigma e_{\mathrm{FE}}\right)\right]=$ \\
\hline $\mathrm{g}_{\mathrm{FG}}\left(\sum e_{F E}\right)$ & Suma ponderada & 1,12 & 0,60 & 0,4 & 0,13 & & \\
\hline & $\beta_{7=} \Sigma\left[g_{\mathrm{FG}}\left(\Sigma e_{F E}\right)\right] / \alpha_{7}$ & & & 0,45 & & & \\
\hline
\end{tabular}

34 Fuente: Elaboración propia. 


\section{CUADRO 12}

Determinación del coeficiente de la categoría $8\left(B_{8}\right)$

\begin{tabular}{|c|c|c|c|c|c|c|c|}
\hline & & & & & & \\
\hline & & \multicolumn{5}{|c|}{ Factores genéricos (peso: $\mathrm{g}_{\mathrm{FC}}$ ) } & \\
\hline & & $\begin{array}{c}\text { Conocimientos } \\
(0,6)\end{array}$ & $\begin{array}{c}\text { Iniciativa } \\
(0,1)\end{array}$ & $\begin{array}{c}\text { Complejidad } \\
(0,2)\end{array}$ & $\begin{array}{c}\text { Responsabilidad } \\
(0,1)\end{array}$ & $\begin{array}{c}\text { Mando / } \\
\text { Dirección (0) }\end{array}$ & \\
\hline \multirow{24}{*}{ 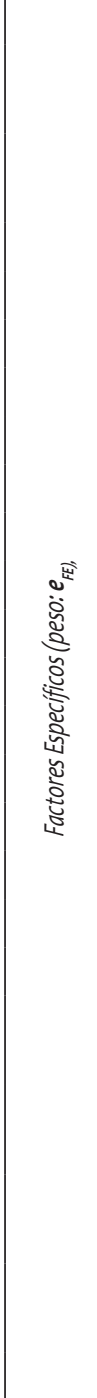 } & Formación básica & 1 & & & & & \\
\hline & $\begin{array}{l}\text { Formación específica } \\
\text { (tcaladva) }\end{array}$ & & & & & & \\
\hline & $\begin{array}{l}\text { Formación especifica } \\
\text { (institucional) }\end{array}$ & & & & & & \\
\hline & Actualización & & & & & & \\
\hline & Requerimientos intelectivos & & & & & & \\
\hline & Especialización profesional & & & & & & \\
\hline & Experiencia previa & & & & & & \\
\hline & Habilidades operativas & & 0,3 & 0,6 & 0,1 & & \\
\hline & Habilidades sociales & & & & & & \\
\hline & Impacto sobre las personas & & & & & & \\
\hline & Impacto sobre la institución & & & & & & \\
\hline & Planificación & & 0,8 & 0,2 & & & \\
\hline & Toma de decisiones & & & & & & \\
\hline & Organización (adva) & & & & & & \\
\hline & Organización (institucional) & & & & & & \\
\hline & Supervisión & & & & & & \\
\hline & Autonomía & & & 0,8 & 0,2 & & \\
\hline & Representación & & & & & & \\
\hline & Coordinación & & & & & & \\
\hline & $\begin{array}{l}\text { Seguimiento de actividades } \\
\text { (admón.) }\end{array}$ & & & & & & \\
\hline & $\begin{array}{l}\text { Seguimiento de actividades } \\
\text { (institucional) }\end{array}$ & & & & & & \\
\hline & $\begin{array}{l}\text { Control de actividades } \\
\text { (admón.) }\end{array}$ & & & & & & \\
\hline & $\begin{array}{l}\text { Control de actividades } \\
\text { (institucional) }\end{array}$ & & & & & & \\
\hline & Tramitación de expedientes & 0,5 & 0,1 & 0,1 & 0,3 & & \\
\hline$\Sigma e_{F E}$ & $\begin{array}{l}\text { Suma de los pesos de } \\
\text { factores específicos }\end{array}$ & 1,5 & 2,1 & 2,6 & 0,8 & & $\Sigma\left[\mathrm{g}_{\mathrm{FG}}\left(\Sigma e_{F E}\right)\right]=$ \\
\hline$g_{F G}\left(\Sigma e_{F E}\right)$ & Suma ponderada & 0,90 & 0,21 & 0,62 & 0,08 & & \\
\hline & $\beta_{8=} \Sigma\left[\mathrm{g}_{\mathrm{FG}}\left(\Sigma e_{F E}\right)\right] / a_{8}$ & & & 0,50 & & & \\
\hline
\end{tabular}




\section{CUADRO 13}

Determinación del coeficiente de la categoría $9\left(b_{q}\right)$

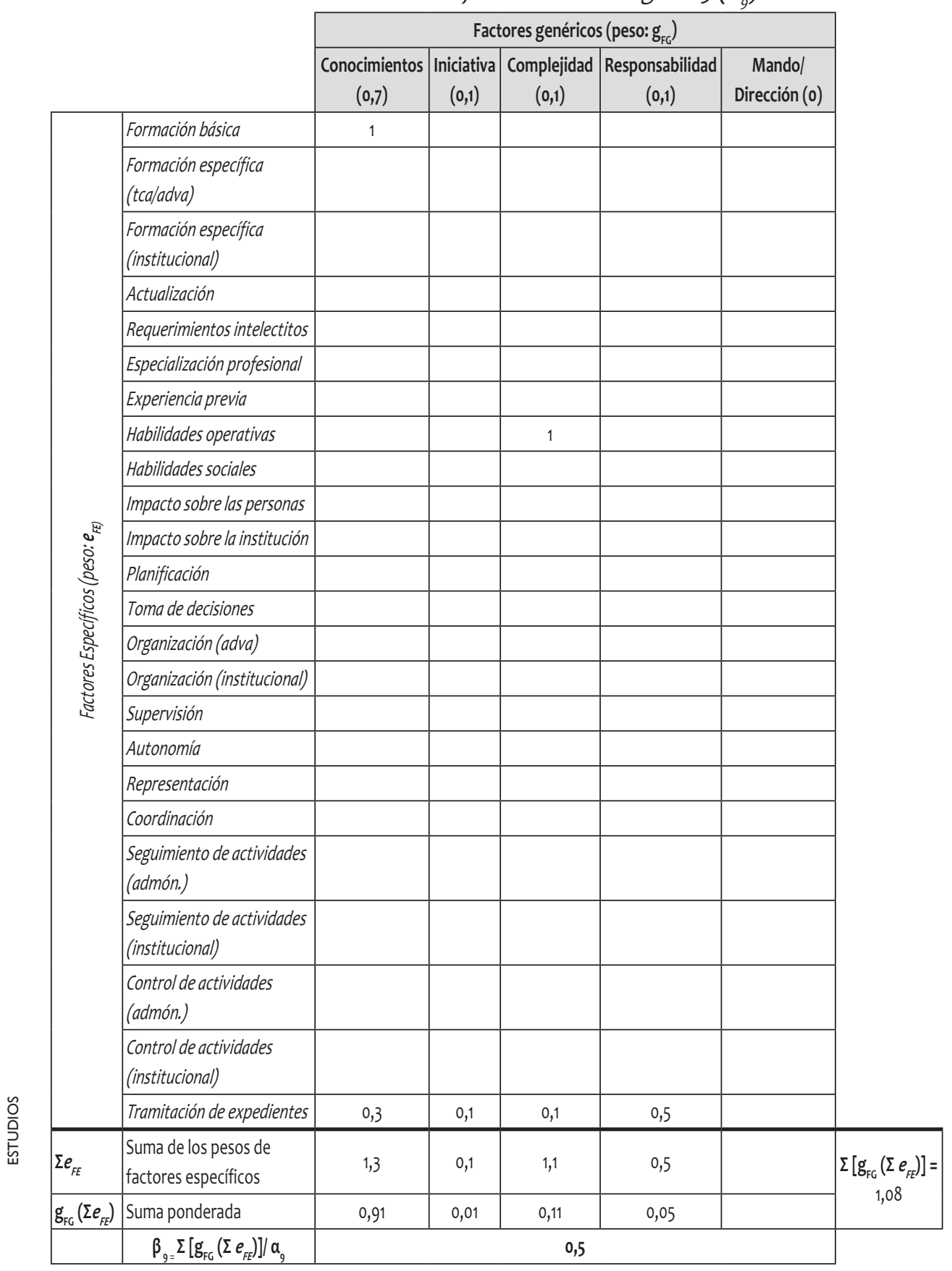

36 Fuente: Elaboración propia. 


\section{CUADRO 14}

Determinación del coeficiente de la categoría $10\left(B_{10}\right)$

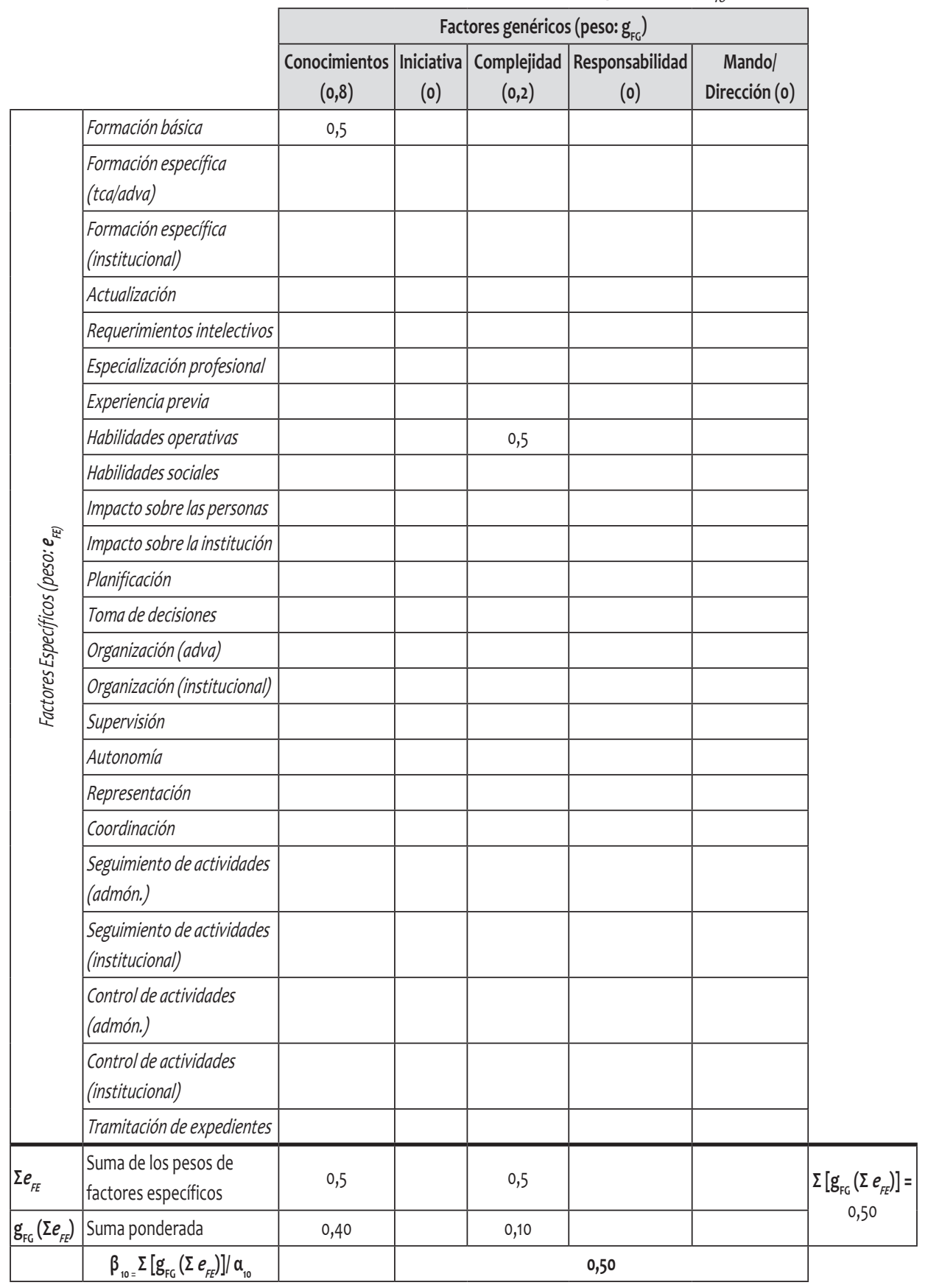


A partir de ahí, para la valoración de un puesto en términos de la categoría (Vcj), habrá que multiplicar el coeficiente $\beta_{\mathrm{i}}$ por la valoración correspondiente al nivel: $V_{c j}=$ $\beta_{\mathrm{j}} \cdot V$ ni. Por ejemplo, para los puestos de la categoría 8 , la valoración es: $V c_{8}=\beta_{8} \cdot V n_{4 ;}$ ya que los puestos de la categoría 8 pertenecen al nivel 4 .

- Coeficiente $\gamma_{p}$ Este coeficiente individualiza los puestos tipo y es la proporción que representa la valoración de las tareas concretas (VTC) de cada puesto tipo con relación al coeficiente $\beta$ j expresado en porcentaje, como se refleja en el cuadro 15.

\section{CUADRO 15}

Coeficiente de puesto $\left(\gamma_{\mathrm{p}}\right)$

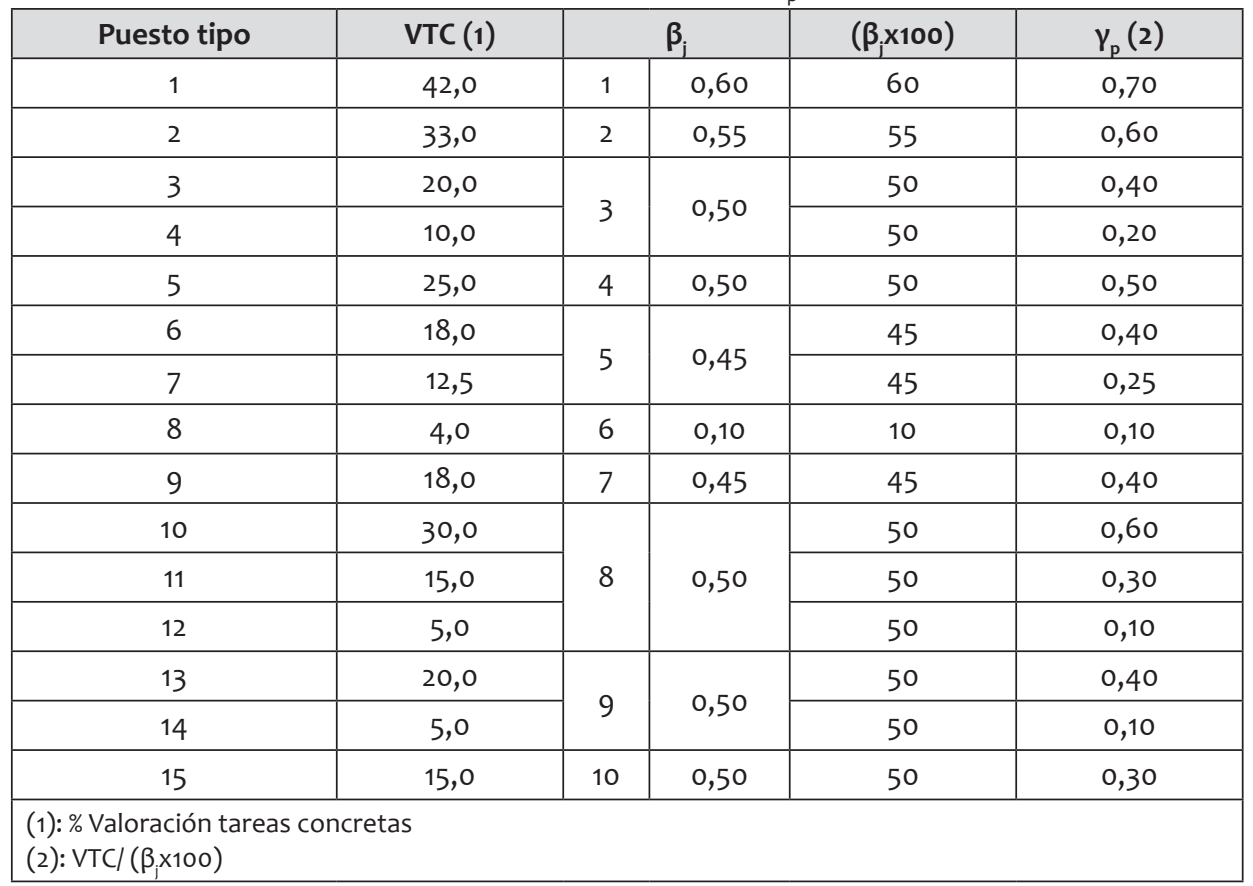

Fuente: Elaboración propia.

Por ejemplo: en la categoría 9 (auxiliar) hay dos puestos tipo (auxiliar de ofimática y auxiliar de servicios generales), identificados por los coeficientes $\gamma_{13}$ y $\gamma_{14}$, respectivamente. Si se analizan las tareas totales asignadas al puesto 13, el 20\% de dichas tareas son específicas del puesto de auxiliar de ofimática. Si se analizan las del puesto 14 , resulta que el $5 \%$ de las tareas son específicas de un puesto de auxiliar de servicios generales. Estas cifras se reflejan en la segunda columna.

Por otra parte, el coeficiente $\beta_{9}$ (categoría a la que pertenecen ambos puestos) 38 etapa anterior. En consecuencia $\gamma_{13}=20 / 50=0,40$ y $\gamma_{14}=5 / 50=0,10$. 
A partir de ahí, la valoración en términos del puesto tipo (Vpy) se obtiene multiplicando el coeficiente $\gamma_{\mathrm{p}}$ por la valoración correspondiente a la categoría: $\mathrm{Vp \gamma}=\gamma_{\mathrm{p}} \mathrm{Vcj}^{8}$.

- Coeficiente $\delta$. Este coeficiente permite cuantificar el último componente del sistema de clasificación profesional; es decir, la carrera en el puesto de trabajo o promoción horizontal.

Esta promoción se articula mediante un sistema de escalones por los que va ascendiendo el trabajador sin necesidad de cambiar de puesto de trabajo, como se verá más adelante.

Para realizar el cálculo de este coeficiente, se parte de que la carrera se hace en la categoría profesional y no en el puesto.

El cálculo no se realiza a partir de formulaciones matemáticas, sino de una estimación de la aportación que la veteranía incorpora en la valoración de puestos, como se puede observar en el cuadro 16.

\section{CUADRO 16}

Coeficiente de carrera

\begin{tabular}{|l|c|c|c|c|c|c|c|c|c|c|}
\hline \multicolumn{1}{|c|}{ Categoría } & $\mathbf{1}$ & $\mathbf{2}$ & $\mathbf{3}$ & $\mathbf{4}$ & $\mathbf{5}$ & $\mathbf{6}$ & $\mathbf{7}$ & $\mathbf{8}$ & $\mathbf{9}$ & $\mathbf{1 0}$ \\
\hline \% Aportación $(\mathrm{A})$ & 1,5 & 1,5 & 1,5 & 1,5 & 1,5 & 1,5 & 1,5 & 1,6 & 1,7 & 1,8 \\
\hline$\delta_{\mathrm{j}}=(\mathrm{A}) \times \beta_{\mathrm{j}}$ & 0,025 & 0,028 & 0,030 & 0,030 & 0,034 & 0,038 & 0,034 & 0,032 & 0,034 & 0,036 \\
\hline
\end{tabular}

Fuente: Elaboración propia.

En este caso, se ha estimado que la veteranía aporta entre un 1,5\% y un 1,8\% de la valoración del puesto en términos de la categoría, cada dos años.

De esta forma, el último componente es la valoración de la carrera horizontal $(V c \delta)$ que se obtendría multiplicando el coeficiente $\delta_{j}$ por la valoración correspondiente a la categoría (Vcj) y por un parámetro "n" del que se tratará más adelante.

Estos son los valores que resultan del análisis de puestos de trabajo con la metodología utilizada en este estudio. Dichos coeficientes tienen carácter atemporal, lo que significa que una vez determinado cada uno de ellos, no tendrían que modificarse anualmente. En su caso, su modificación procederá cuando se utilicen otros métodos de valoración de puestos, otros criterios u otros factores genéricos y específicos distintos a los utilizados aquí.

\section{EL SISTEMA DE PROMOCION PROFESIONAL}

El sistema que se contempla para el personal laboral de esta institución comprende dos tipos de promoción: horizontal y vertical.

8 Así, para el puesto tipo $13, V p_{13}=\gamma_{13} . c_{9}$ y para el 14; $V p_{14}=\gamma_{14} . c_{9}$ 


\section{Promoción horizontal}

Es la carrera profesional en el puesto de trabajo, bajo la premisa de que el empleado "enriquece" el puesto (desarrolla mejor las tareas) mediante la experiencia, la formación y el desempeño. Ese "enriquecimiento" conlleva que pueda desarrollar una progresión profesional "horizontal" mediante el reconocimiento de diferentes "tramos" o "estadios" en su desarrollo y se articula en un sistema de escalones de ascenso que serán consecutivos y no conlleva cambio de categoría profesional ni de puesto de trabajo9.

Este tipo de promoción se contempla dentro del sistema a través del parámetro “ $n$ ” y del coeficiente $\delta$. El primero representa el escalón o tramo en el que se encuentra el empleado por su permanencia en la institución y adquiere valores enteros consecutivos cada dos años a partir del valor inicial de cero, de forma que cada escalón tiene dos subescalones o peldaños, tal como se ilustra en el siguiente cuadro:

\section{CUADRO 17}

Escalones de la promoción horizontal

\begin{tabular}{|l|l|l|l|l|l|l|l|l|l|l|l|l|l|l|l|l|l|}
\hline \multicolumn{10}{|c|}{$\mathbf{n}$} & \multicolumn{10}{|c|}{$\mathbf{0}$} & \multicolumn{2}{|c|}{$\mathbf{1}$} & \multicolumn{2}{|c|}{2} & \multicolumn{2}{|c|}{3} & \multicolumn{2}{|c|}{4} & \multicolumn{2}{|c|}{5} & \multicolumn{3}{|c|}{6} & 7 \\
\hline Subescalón & $1^{\circ}$ & $2^{\circ}$ & $1^{\circ}$ & $2^{\circ}$ & $1^{\circ}$ & $2^{\circ}$ & $1^{\circ}$ & $2^{\circ}$ & $1^{\circ}$ & $2^{\circ}$ & $1^{\circ}$ & $2^{\circ}$ & $1^{\circ}$ & $2^{\circ}$ & $1^{\circ}$ & $2^{\circ}$ \\
\hline Año & 1 & 2 & 3 & 4 & 5 & 6 & 7 & 8 & 9 & 10 & 11 & 12 & 13 & 14 & 15 & 16 \\
\hline
\end{tabular}

Fuente: Elaboración propia.

De esta forma, por ejemplo, un empleado que tenga una antigüedad de siete años en el mismo puesto de trabajo desde su ingreso en la institución, se encuentra en el escalón $n=3$, subescalón primero.

Así pues, "n" es el parámetro que individualiza la posición de cada trabajador en el sistema y su progresión está condicionada a la valoración positiva basada en el desarrollo de las competencias y en el rendimiento.

En este punto conviene señalar que para poder implantar esta modalidad de carrera profesional horizontal se deberá:

- Valorar la trayectoria y actuación profesional, la calidad de los trabajos realizados, los conocimientos adquiridos y el resultado de la evaluación del desempeño.

9 Este modelo está inspirado en el vigente en la Unión Europea, regulado en el Reglamento $\mathrm{N}^{\circ} 31$ (CEE) 11 (CEEA) por el que se establece el Estatuto de los funcionarios y el régimen aplicable a los otros agentes de la Comunidad Económica Europea y de la Comunidad Europea de la Energía Atómica y modificaciones posteriores. 
- Establecer los efectos de la evaluación en la carrera profesional horizontal y en la percepción de las retribuciones complementarias previstas en el art. 24 del EBEP'

\section{Promoción vertical}

Es la que tiene lugar cuando un empleado pasa a ocupar un puesto de trabajo superior en el cuadro de clasificación profesional ${ }^{11}$ y puede darse desde un puesto a otro que pertenezca:

a) Al mismo grupo, nivel y categoría: es una promoción intracategoría en la que solo tiene lugar el cambio de coeficiente de puesto $\gamma$.

b) Al mismo grupo, mismo nivel y distinta categoría: es una promoción intercategoría en la que cambian un grado los coeficientes de puesto $(\gamma)$, categoría $(\beta)$ y carrera horizontal $(\delta)$.

c) Al mismo grupo, distinto nivel y distinta categoría: es una promoción internivel en la que cambian un grado los coeficientes de puesto $(\gamma)$, categoría $(\beta)$, nivel $(\alpha)$ y carrera administrativa $(\delta)$.

d) A distintos grupo, nivel y categoría: es una promoción intergrupo en la que cambian un grado los coeficientes de puesto $(\gamma)$, categoría $(\beta)$, nivel $(\alpha)$ y carrera administrativa $(\delta)$.

\section{Relación entre la promoción vertical y la horizontal}

Una vez establecidas la promoción horizontal y las modalidades de promoción vertical, en las que tienen lugar cambios en los coeficientes de clasificación, procede atender al ámbito individual del empleado; es decir, a su concreta posición en el sistema de clasificación, con motivo de la promoción vertical. Este aspecto viene determinado por el parámetro "n".

Se trata de elaborar un sistema justo y equitativo, estableciendo el escalón concreto en el que debe situarse el empleado promocionado.

Por una parte, es evidente que quien promociona a un puesto superior en el cuadro clasificatorio no debería ocupar el escalón cero en el nuevo puesto, porque ello lo

10 Con estas premisas, la carrera profesional horizontal debe reunir las siguientes características: 1) Objetivable y mensurable y 2) Evaluable por una Comisión de evaluación establecida al efecto.

11 Este aspecto ha de regularse en el sistema de cobertura y provisión de vacantes, que debe establecer las condiciones y requisitos que han de cumplir los candidatos, así como los procesos selectivos correspondientes. 
equipararía a otro que hubiera ingresado en la institución por turno libre en dicho puesto, lo cual supondría ignorar su propia trayectoria profesional en la institución.

Por otra parte, también es evidente que el promocionado no debería ocupar el mismo escalón que tiene en el puesto de origen porque ello lo equipararía a todos los compañeros del nuevo puesto que se encuentren en el mismo escalón ignorando, en este caso, la trayectoria profesional de estos en relación con el ascendido.

Estas dos consideraciones llevan a proponer un sistema que trata de conciliar esas dos posiciones y que se visualiza en el gráfico 2. Según la modalidad, el trabajador promocionado se sitúa en un escalón inferior en uno, dos o tres grados al que tenía en la fecha de la promoción, y el tiempo de permanencia en el de procedencia computa en el nuevo, salvo para la promoción intergrupo. Se presentan así las siguientes variantes de promoción:

- Promoción intracategoría: el trabajador promocionado se sitúa en un escalón inferior en un grado y el tiempo de permanencia en el escalón de procedencia computa en el nuevo ${ }^{12}$.

- Promoción intercategoría: el trabajador promocionado a la nueva categoría se situaría en dos escalones anteriores y el tiempo de permanencia en el escalón de procedencia computa en el nuevo ${ }^{13}$.

- Promoción internivel: el trabajador promocionado al nuevo nivel se sitúa en tres escalones inferiores y el tiempo de permanencia en el escalón de procedencia computa en el nuevo ${ }^{14}$.

- Promoción intergrupo: el trabajador promocionado al nuevo grupo se sitúa en cuatro escalones inferiores, no computándosele el tiempo de permanencia en el escalón de procedencia ${ }^{15}$.

12 Por ejemplo, si un Auxiliar de Servicios Generales que se encuentre en el escalón 4 (Código: IV.5.9.14.5.4) promociona a un puesto de Auxiliar de Ofimática, se colocaría en el escalón 3 (Código: IV.5.9.14.5.3), computándosele el tiempo de permanencia en el escalón de origen.

13 Por ejemplo, si un Asesor de Unidad que se encuentre en el escalón 4 (Código: I.2.6.8.6.4) promociona a un puesto de Asesor Coordinador de Unidad, se colocaría en el escalón 2 (Código: I.2.6.7.6.2). El tratamiento en cuanto al tiempo computable en el nuevo puesto es el mismo que en el supuesto anterior.

14 Por ejemplo, si un Asesor Coordinador que se encuentre en el escalón 4 (Código: I.2.4·5.4.4) promociona a un puesto de Jefe de Departamento, se colocaría en el escalón 1 (Código: I.1.3.4.3.1). El tratamiento en cuanto al tiempo computable en el nuevo puesto es el mismo que en los supuestos anteriores.

15 Por ejemplo, si un Técnico de Gestión que se encuentre en el escalón 4 (Código: II.3.7.9.7.4) promociona a un puesto de Técnico Superior, se colocaría en el escalón 1 (Código: I.2.6.8.6.1). Esto significa

42 que si el trabajador promocionado tenía, por ejemplo, 20 meses de permanencia en el escalón 4, este 
GRÁFICO 2

Representación gráfica de la relación entre la promoción horizontal y la vertical

\begin{tabular}{|c|c|c|c|c|c|c|c|c|c|c|}
\hline Peldaño & & & \multicolumn{2}{|c|}{$n-3$} & \multicolumn{2}{|c|}{$n-2$} & \multicolumn{2}{|c|}{$\mathrm{n}-\mathbf{1}$} & \multicolumn{2}{|c|}{$\mathbf{n}$} \\
\hline Escalón & & & $1^{\circ}$ & $2^{\circ}$ & $1^{\circ}$ & $2^{\circ}$ & $1^{\circ}$ & $2^{\circ}$ & $1^{\circ}$ & $2^{\circ}$ \\
\hline \multirow{2}{*}{ Intracategoría } & $\begin{array}{l}\text { Puesto tras la } \\
\text { promoción }\end{array}$ & $\alpha_{i} \beta_{j} \gamma_{p-1} \delta_{j} n-1$ & & & & & & & & \\
\hline & $\begin{array}{c}\text { Puesto de } \\
\text { origen }\end{array}$ & $a_{i} \beta_{j} \gamma_{p} \delta_{j} n$ & & & & & & & & \\
\hline \multirow{2}{*}{ Intercategoría } & $\begin{array}{l}\text { Puesto tras la } \\
\text { promoción }\end{array}$ & $a_{i} \beta_{j-1} \gamma_{p-1} \delta_{j-1} n-2$ & & & & & & & & \\
\hline & $\begin{array}{l}\text { Puesto de } \\
\text { origen }\end{array}$ & $a_{i} \beta_{j} \gamma_{p} \delta_{j} n$ & & & & & & & & \\
\hline \multirow{2}{*}{ Internivel } & $\begin{array}{l}\text { Puesto tras la } \\
\text { promoción }\end{array}$ & $\alpha_{i-1} \beta_{j-1} \gamma_{p-1} \delta_{j-1} n-3$ & & & & & & & & \\
\hline & $\begin{array}{c}\text { Puesto de } \\
\text { origen }\end{array}$ & $a_{i} \beta_{j} \gamma_{p} \delta_{i} n$ & & & & & & & & \\
\hline \multirow{2}{*}{ Intergrupo } & $\begin{array}{l}\text { Puesto tras la } \\
\text { promoción }\end{array}$ & $\alpha_{i-1} \beta_{j-1} \gamma_{p-1} \delta_{j-1} n-3$ & & & & & & & & \\
\hline & $\begin{array}{l}\text { Puesto de } \\
\text { origen }\end{array}$ & $a_{i} \beta_{j} \gamma_{p} \delta_{j} n$ & & & & & & & & \\
\hline
\end{tabular}

Fuente: Elaboración propia.

\section{EL SISTEMA RETRIBUTIVO}

El sistema retributivo se encuentra asociado a la estructura de clasificación profesional, de forma que cada puesto tenga asignadas unas retribuciones articuladas en función de los coeficientes de clasificación.

El procedimiento para la elaboración de la tabla salarial consiste en:

a) Formular una ecuación salarial utilizando los valores de los coeficientes de clasificación en la que todas las retribuciones, básicas y complementarias, aparecen referenciadas a un parámetro "B".

b) Elaborar el cuadro retributivo general a partir de la ecuación salarial en función del parámetro "B" y la tabla salarial en términos monetarios.

\section{a) La ecuación salarial}

La valoración total (VT) de un puesto de trabajo, en términos de los coeficientes de clasificación, se hace sumando los componentes de nivel (Vni), categoría (Vc), puesto tipo (Vp) y carrera horizontal $(\mathrm{Vc} \delta)$ :

$$
V T=V n i+V c j+V p \gamma+V c \delta
$$


Teniendo en cuenta que:

$$
\begin{aligned}
& V n i=(1+x)^{a i} \\
& V c j=\beta_{j .} V_{=} \beta_{j}(1+x)^{a i} \\
& V p \gamma=\gamma_{p .} V c=\gamma_{p .} \beta_{j}(1+x)^{a i} \\
& V c \delta=n \delta_{j} . V p=n \delta_{j .} \gamma_{p .} \beta_{j}(1+x)^{a i}
\end{aligned}
$$

La suma de esos componentes es:

$$
V T=(1+x)^{a i}\left[1+\beta_{j}\left(1+\gamma_{p}+n . \delta_{j}\right)\right]
$$

Aplicando, en esa expresión, los valores de los coeficientes de todos los puestos tipo se obtiene la tabla de valoraciones que se presenta en el cuadro 18 , donde se puede observar que la última columna refleja la valoración total de cada puesto en función del escalón "n”.

Ahora bien, al tratarse de una expresión adimensional basada en coeficientes, es necesario disponer de un conversor que permita expresarla en términos monetarios. Este conversor es el parámetro B. Así, las retribuciones totales (RT) de cada puesto tipo vienen dadas por la expresión $\mathrm{RT}=\mathrm{B} \times \mathrm{VT}$, que es la ecuación salarial.

$$
R T=B \cdot(1+x)^{a i}\left[1+\beta_{j}\left(1+\gamma_{p}+n \delta_{j}\right)\right]
$$

El conversor B se calcula a partir del montante de masa salarial disponible (MS) y de la suma las valoraciones de todos y cada uno de los puestos de trabajo ${ }^{16}$ : $B=M S / \Sigma V T T$

\section{b) Cuadro retributivo y tabla salarial}

Si el valor contenido en cada una de las celdas del cuadro 18 , se multiplica por el conversor "B", se obtiene el cuadro retributivo de la institución.

La representación gráfica se refleja en el gráfico 3 en donde se han representado las retribuciones totales para los cinco primeros escalones, desde $n=0$ hasta $n=4$, para un valor de B de 9.800 .

16 Ejemplo: si se dispone de una masa salarial de 1.901.200 euros y la suma de
44 todos los puestos de trabajo de la institución es 194 el valor de B es: B = 9.800 euros. 


\section{CUADRO 18}

Valoración total de los puestos

\begin{tabular}{|c|c|c|c|c|c|c|c|}
\hline Nivel & Categoría & Puesto tipo & $\begin{array}{l}\text { Nivel Vni = } \\
\quad(1+x)^{a}\end{array}$ & $\begin{array}{l}\text { Categoría } \\
\text { Vcj }=\beta_{\mathrm{j}} \text {. Vni }\end{array}$ & $\begin{array}{c}\text { Pto tipo } \\
V p y= \\
\gamma_{p} \cdot V c j\end{array}$ & $\begin{array}{l}\text { Carrera Vco } \\
=\delta_{j} n \cdot V c j\end{array}$ & TOTAL \\
\hline \multirow{4}{*}{$\begin{array}{c}1 \\
a_{1}=7,973\end{array}$} & $\begin{array}{c}1 \\
\beta_{1}=0,60 \\
\delta_{1}=0,025\end{array}$ & $\begin{array}{c}1 \\
\gamma_{1}=0,70\end{array}$ & 3,4180 & 2,0508 & 1,4355 & $0,1367 \cdot n$ & $6,9043+0,1367 \cdot n$ \\
\hline & $\begin{array}{c}2 \\
\beta_{2}=0,55 \\
\delta_{2}=0,028\end{array}$ & $\begin{array}{c}2 \\
\gamma_{2}=0,60\end{array}$ & 3,4180 & 1,8799 & 1,1279 & $0,1483 \cdot n$ & $6,4258+0,1483 \cdot n$ \\
\hline & \multirow{2}{*}{$\begin{array}{c}3 \\
\beta_{3}=0,50 \\
\delta_{3}=0,030\end{array}$} & $\begin{array}{c}3 \\
V_{3}=0,40\end{array}$ & 3,4180 & 1,7090 & 0,6836 & $0,1538 . n$ & $5,8105+0,1538 . n$ \\
\hline & & $\begin{array}{c}4 \\
\gamma_{4}=0,20\end{array}$ & 3,4180 & 1,7090 & 0,3418 & $0,1538 . n$ & $5,4687+0,1538 \cdot n$ \\
\hline \multirow{4}{*}{$\begin{array}{c}2 \\
a_{2}=6,525\end{array}$} & $\begin{array}{c}4 \\
\beta_{4}=0,50 \\
\delta_{4}=0,030\end{array}$ & $\begin{array}{c}5 \\
\gamma_{5}=0,50\end{array}$ & 2,7342 & 1,3671 & 0,6835 & $0,1230 . n$ & $4,7848+0,1230 . n$ \\
\hline & $\begin{array}{c}5 \\
\beta_{5}=0,45 \\
\delta_{5}=0,034\end{array}$ & $\begin{array}{c}6 \\
v_{6}=0,40\end{array}$ & 2,7342 & 1,2304 & 0,4922 & $0,1348 . n$ & $4,4567+0,1348 \cdot n$ \\
\hline & \multirow{2}{*}{$\begin{array}{c}6 \\
\beta_{6}=0,40 \\
\delta_{6}=0,038\end{array}$} & $\begin{array}{c}7 \\
\gamma_{7}=0,25\end{array}$ & 2,7342 & 1,0937 & 0,2734 & $0,1455 \cdot n$ & $4,1013+0,1455 \cdot n$ \\
\hline & & $\begin{array}{c}8 \\
\gamma_{8}=0,10\end{array}$ & 2,7342 & 1,0937 & 0,1094 & $0,1455 \cdot n$ & $3,9372+0,1455 \cdot n$ \\
\hline $\begin{array}{c}3 \\
a_{3}=5,078\end{array}$ & $\begin{array}{c}7 \\
\beta_{7}=0,45 \\
\delta_{7}=0,034\end{array}$ & $\begin{array}{c}9 \\
\gamma_{9}=0,40\end{array}$ & 2,1875 & 0,9844 & 0,3938 & $0,1078 . n$ & $3,5657+0,1078 \cdot n$ \\
\hline \multirow{3}{*}{$\begin{array}{c}4 \\
a_{4}=3,630\end{array}$} & \multirow{3}{*}{$\begin{array}{c}8 \\
\beta_{8}=0,50 \\
\delta_{8}=0,032\end{array}$} & $\begin{array}{c}10 \\
\gamma_{10}=0,60\end{array}$ & 1,7499 & 0,8750 & 0,5250 & $0,0840 . n$ & $3,1498+0,0840 \cdot n$ \\
\hline & & $\begin{array}{c}11 \\
\gamma_{11}=0,30\end{array}$ & 1,7499 & 0,8750 & 0,2625 & $0,0840 . n$ & $2,8874+0,0840 . n$ \\
\hline & & $\begin{array}{c}12 \\
\gamma_{12}=0,10\end{array}$ & 1,7499 & 0,8750 & 0,0875 & $0,0840 . n$ & $2,7124+0,0840 . n$ \\
\hline \multirow{2}{*}{$\begin{array}{c}5 \\
a_{5}=2,183\end{array}$} & \multirow{2}{*}{$\begin{array}{c}9 \\
\beta_{9}=0,50 \\
\delta_{9}=0,034\end{array}$} & $\begin{array}{c}13 \\
\gamma_{13}=0,40\end{array}$ & 1,4001 & 0,7000 & 0,2800 & $0,0714 . n$ & $2,3801+0,0714 \cdot n$ \\
\hline & & $\begin{array}{c}14 \\
\gamma_{14}=0,10\end{array}$ & 1,4001 & 0,7000 & 0,0700 & $0,0714 . n$ & $2,1701+0,0714 \cdot n$ \\
\hline $\begin{array}{c}6 \\
a_{6}=1\end{array}$ & $\begin{array}{c}10 \\
\beta_{10}=0,50 \\
\delta_{10}=0,036\end{array}$ & $\begin{array}{c}15 \\
\gamma_{15}=0,30\end{array}$ & 1,1667 & 0,5833 & 0,1750 & $0,0630 . n$ & $1,9250+0,0630 . n$ \\
\hline
\end{tabular}

Fuente: Elaboración propia. 
GRÁFICO 3

Retribuciones totales de los puestos tipo para $B=9,800$

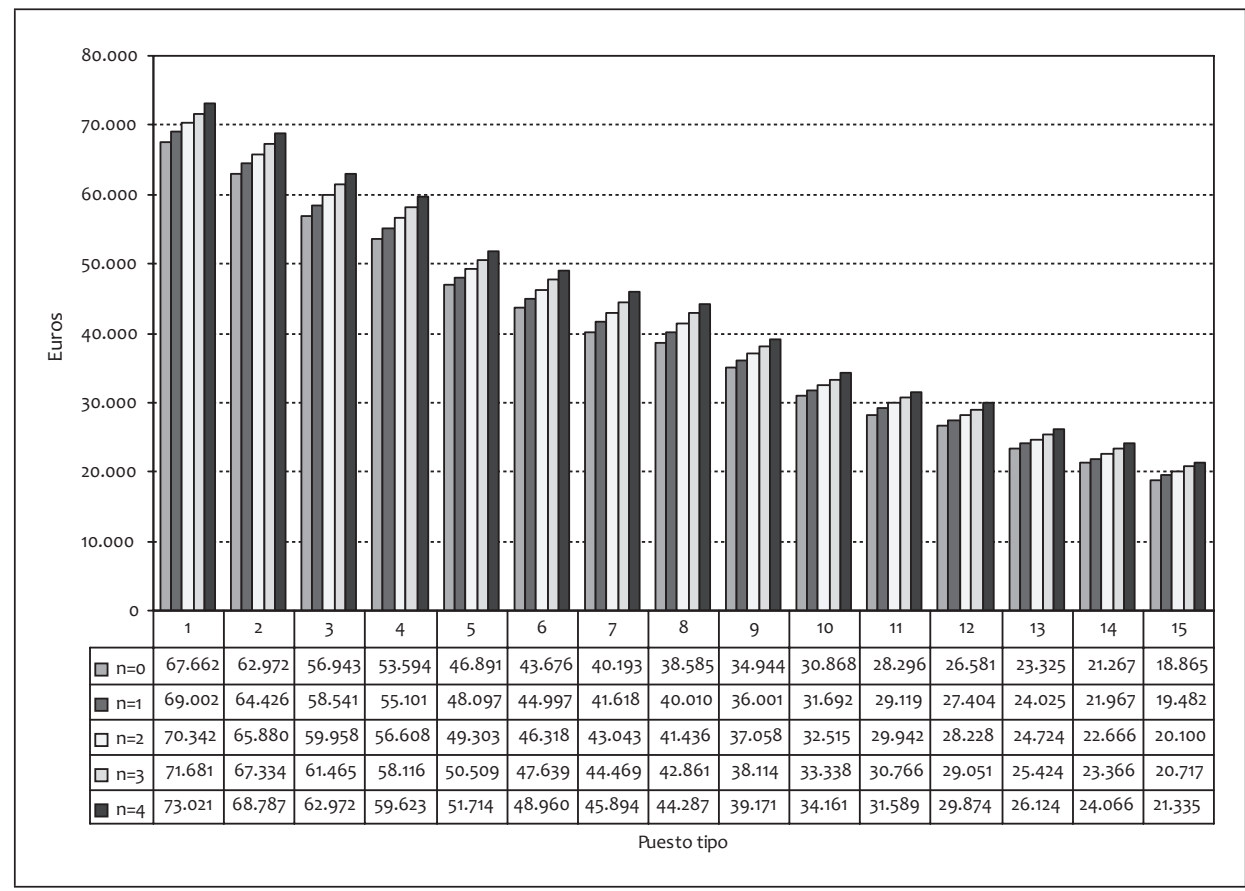

Fuente: Elaboración propia.

Se puede observar que la carrera horizontal permite que un empleado situado en un escalón alto pueda tener unas retribuciones totales iguales, o mayores, que otro que ocupe un puesto superior pero en un escalón inferior ${ }^{17}$.

La tabla salarial de cada año se obtendría multiplicando por el valor de B, todos los componentes retributivos reflejados en el cuadro 17.

De esta forma, cualquier empleado podría calcular sus retribuciones totales y parciales anuales con sólo conocer el valor de B.

\section{CONCLUSIONES}

El EBEP proporciona el marco general para la gestión de recursos humanos en las administraciones públicas. Este es el punto de partida del estudio realizado en este trabajo que tiene por objeto diseñar los sistemas de clasificación profesional,

17 Por ejemplo, la retribución del puesto tipo 14 en el escalón $n=3$ (23.366€) es algo superior a la del puesto tipo 13 en el escalón $n=0(23.325 €)$. 
carrera profesional y de retribuciones para el personal laboral que presta servicios en un organismo público y que tiene una plantilla de cien empleados.

Se aborda la cuestión de una manera integral, de forma que se puedan establecer y regular las interconexiones existentes entre los tres sistemas mencionados que son también aspectos nucleares de cualquier modelo integral de gestión de recursos humanos.

A partir de la información disponible en los documentos de trabajo, se diseña el sistema de clasificación profesional mediante la ordenación de los puestos, atendiendo a los niveles de titulación, formación y capacitación para ejercer los cometidos de los distintos niveles de la prestación del servicio.

Se aplica una metodología inspirada en la técnica SAO (Sistema de Análisis Organizacional) que permite obtener quince puestos tipo, los cuales se clasifican y ordenan en cuatro grupos, seis niveles y diez categorías profesionales.

Los puestos tipo se codifican y valoran utilizando una técnica de valoración mediante la asignación y cuantificación de coeficientes que tienen carácter atemporal.

Se formulan cinco coeficientes de clasificación: de nivel, de categoría, de puesto tipo y de carrera. Este último permite articular el sistema de promoción profesional.

En lo que respecta al sistema de promoción y carrera administrativa, el EBEP ha incorporado una regulación innovadora y novedosa al permitir que se articulen modelos de carrera horizontal desvinculada de los cambios de puesto de trabajo y basada en el desarrollo de las competencias y en el rendimiento, de forma que la carrera pueda proyectarse sobre dos aspectos: una mejor retribución y una mayor cualificación del trabajo. Con el marco general establecido en el Estatuto, el sistema que se contempla comprende dos tipos de promoción: horizontal y vertical.

- Promoción horizontal: es la carrera profesional en el puesto de trabajo, bajo la premisa de que el empleado "enriquece" el puesto mediante la experiencia, la formación y el desempeño. Ese "enriquecimiento" conlleva que pueda desarrollar una progresión profesional "horizontal" mediante el reconocimiento de diferentes "tramos" o "estadios" en su desarrollo. (De esta forma, la progresión a escalones superiores de cada empleado está condicionada por la valoración positiva obtenida a partir de la evaluación del desempeño, cuyo procedimiento también tiene que establecerse y someterse a las previsiones del art. 24 del EBEP).

- Promoción vertical: es la que tiene lugar cuando un empleado pasa a ocupar un puesto de trabajo superior en el cuadro de clasificación profesional y puede adoptar cuatro modalidades: intracategoría, intercategoría, internivel e intergrupo. 
Una vez establecidas y definidas la promoción horizontal y las modalidades de promoción vertical, se diseña, también, el modelo que interconecta a ambas; es decir el escalón concreto de carrera horizontal en el que debe situarse el empleado que experimente una promoción vertical.

El sistema retributivo que se propone se encuentra asociado a la estructura de clasificación profesional, de forma que cada puesto tiene asignadas unas retribuciones articuladas en función de los coeficientes asociados al mismo.

Las retribuciones asociadas al grupo y nivel crecen en progresión geométrica, las asociadas a la categoría, para cada nivel, así como las asociadas al puesto y a la carrera administrativa crecen en progresión aritmética. Ello da lugar a una ecuación salarial que se aplica a cada empleado en función de su posición en el cuadro de clasificación y del escalón de carrera en el que se encuentre.

Con este modelo, la carrera horizontal permite que un empleado situado en un escalón alto pueda tener unas retribuciones totales iguales o mayores que otro que ocupe un puesto superior pero en un escalón inferior.

Finalmente, hay que hacer hincapié en el sistema de evaluación del desempeño, el cual va a exigir el desarrollo en las organizaciones públicas de importantes capacidades internas: sistemas de definición de objetivos, indicadores de rendimiento, instrumentos para valorar competencias, técnicos especializados así como directivos capacitados y comprometidos.

\section{REFERENCIAS BIBLIOGRÁFICAS Y DOCUMENTALES}

Alia Ramos, M. y Montane Merinero, M.J. (1995), Clasificación profesional y movilidad en el trabajo. Valencia: CISS.

Cantero Martínez, J. (2007), “Ámbito de aplicación y clases de personal al servicio de las Administraciones Públicas”, en L. Ortega Álvarez, dir., Comentarios al Estatuto Básico del Empleado Público. Madrid: La Ley y El Consultor de los Ayuntamientos.

Castillo Mendoza, C.A. (1990), "Control y organización capitalista del trabajo. El estado de la cuestión”, Sociología del Trabajo, 9: 117-140.

Chiavenato, I. (1999), Introducción a la teoría General de las Administración. México D.F.: McGraw Hill.

Cruz Villalón, J. (1983), Las modificaciones de la prestación de trabajo. Madrid: Ministerio de Trabajo y Seguridad Social.

Edwards, P. (1990), El conflicto en el trabajo. Madrid: Ministerio de Trabajo y Seguridad Social.

Férez Fernández, M. (2006), La carrera administrativa: nuevas perspectivas. Estudios de Relaciones Laborales. Barcelona: CEMICAL.

Fernández-Ríos, M. y Sánchez, J.C. (1997), Valoración de
mentos, métodos y ejercicios. Madrid: Díaz de Santos. 
Francis, G. y Holloway, J. (2002), "Beyond comparisons - the role for the operational researcher in benchmarking", Journal of the Operational Research Society, 53: 283-291.

Godino Reyes M. (1996), El contrato de trabajo en la Administración Pública. Madrid: Civitas-Argentaria.

Koontz, H. y Weihrich, H. (1990), Administración. México D.F.: McGraw Hill.

Landa Zapirain, J. P. (2008), "El empleo público después del Estatuto Básico del Empleado Público: en particular sus aspectos colectivos." XIX Jornades Catalanes de Dret Social. Barcelona.

Manzana Laguarda, R. (2006), Derechos y deberes del funcionario. Valencia: Tirant lo Blanch.

Milgrom, P. Y Roberts, J. (1992), Economics, Organization and Management. New Jersey: Prentice Hall.

Milkovich, G.T. y Newman, J.M. (1996), Compensation. Chicago: Irwin.

Palomar Olmeda, A. (2007), "Derecho a la carrera y promoción interna", en Comentarios a la Ley del Estatuto Básico del Empleado Público. Valladolid: Lex Nova.

Ramió, C. (2002), Teoría de la organización y administración pública. Madrid: Tecnos.

Sala Franco, T. y López Tarruella Martínez, F. (1991), La modificación de la prestación de trabajo. Bilbao: Deusto.

Sala Franco, T. (1998), "Movilidad funcional”, en Comentarios a las leyes laborales. El Estatuto de los Trabajadores, Tomo VIII. Madrid: Edersa.

Sala Franco, T. (2007), “El personal laboral. La relación laboral especial de empleo público”, en M. Sánchez Morón, ed., Comentarios a la Ley del Estatuto Básico del Empleado Público. Valladolid: Lex Nova.

Stoner, J.F. et al. (1996), Administración. México D.F.: Prentice Hall. 